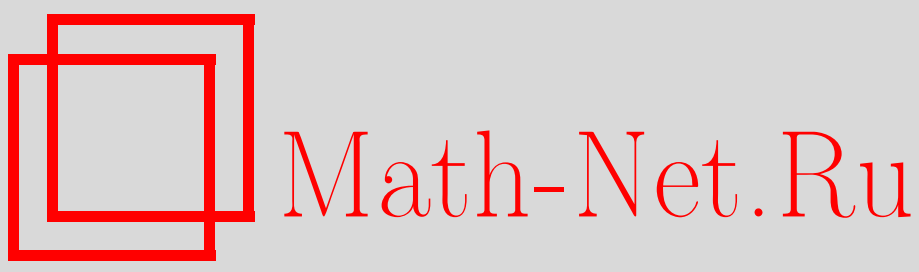

С. Г. Танкеев, О стандартной гипотезе для комплексных абелевых схем над гладкими проективными кривыми, Изв. РАН. Сер. матем., 2003, том 67, выпуск 3, 183224

DOI: https://doi.org/10.4213/im439

Использование Общероссийского математического портала Math-Net.Ru подразумевает, что вы прочитали и согласны с пользовательским соглашением

http://www.mathnet.ru/rus/agreement

Параметры загрузки:

IP : 54.198 .55 .26

26 апреля 2023 г., $17: 52: 56$ 
УДК 512.6

\author{
С.Г. Танкеев
}

\title{
О стандартной гипотезе для комплексных абелевых схем над гладкими проективными кривыми
}

\author{
К 80-летию Игоря Ростиславовича Шафаревича
}

\begin{abstract}
Гипотеза Ходжа для абелевых многообразий сведена к вопросу о существовании алгебраического изоморфизма $H^{2}\left(C, R^{2 d-i} \pi_{*} \mathbb{Q}\right) \stackrel{\sim}{\rightarrow} H^{0}\left(C, R^{i} \pi_{*} \mathbb{Q}\right)$ для всех $i \geqslant 2$ и для всех комплексных абелевых схем с главной поляризацией $\pi: X \rightarrow C$ относительной размерности $d$ над гладкими проективными кривыми. Если канонически определенные циклы Ходжа $\alpha_{i}(X / C) \in$ $H^{0}\left(C, R^{i} \pi_{*} \mathbb{Q}\right) \otimes H^{0}\left(C, R^{i} \pi_{*} \mathbb{Q}\right)$ алгебраичны для всех натуральных чисел $i \geqslant 2$, то стандартная гипотеза Гротендика $B(X)$ об алгебраичности операторов $\Lambda$ и $*$ верна для $X$. Доказана гипотеза $B(X)$ для абелевой схемы при условии, что $\operatorname{End}\left(X_{s}\right)=\mathbb{Z}$ для некоторого геометрического слоя $X_{s}$ неисключительной размерности.
\end{abstract}

Библиография: 34 наименования.

\section{Введение}

Пусть $X$ - гладкое проективное $d$-мерное многообразие над полем комплексных чисел $\mathbb{C}$. Выбор $\sqrt{-1} \in \mathbb{C}$ определяет изоморфизм $\mathbb{Q}(1) \simeq \mathbb{Q}$. Обозначим через $\operatorname{cl}_{X}(Z) \in H^{2 i}(X, \mathbb{Q})$ класс алгебраического цикла $Z$ коразмерности $i$ на $X$.

ГИПотеЗА ХоДЖА $\left(\operatorname{Hodge}^{i}(X)[13]\right)$. Для данного натурального числа $i$ $\mathbb{Q}$-пространство $H^{2 i}(X, \mathbb{Q}) \cap H^{i, i}(X, \mathbb{C})$ порождается классами алгебраических ииклов коразмерности $i$ на $X$.

Если гипотеза $\operatorname{Hodge}^{i}(X)$ верна для любого натурального числа $i$, то гипотеза Ходжа $\operatorname{Hodge}(X)$ выполняется для $X$. По теореме Лефшеца $\operatorname{Hodge}^{1}(X)$ верна.

В обзорах $[27]$ и $[10]$ дано введение в гипотезу Ходжа для многообразий Ферма и для абелевых многоообразий.

Напомним, что линейное отображение $u^{*}: H^{i}(X, \mathbb{Q}) \rightarrow H^{j}(X, \mathbb{Q})$ называется алгебраическим, если оно является ограничением отображения $H^{*}(X, \mathbb{Q}) \rightarrow$ $H^{*}(X, \mathbb{Q})$, индуцированного элементом $\mathbb{Q}$-векторного пространства, порожденного алгебраическими классами $\left[14\right.$, п. 1.3.6]. Другими словами, $u^{*}$ алгебраично, если существует такой алгебраический цикл $Z$ с рациональными коэффициентами,

Работа выполнена при финансовой поддержке Российского фонда фундаментальных исследований (грант № 00-01-00058), при частичной поддержке Института высших научных исследований (Бюр-сюр-Иветт, декабрь 2000 г.) и Математического института Макса Планка (Бонн, февраль-май 2001 г.). Автор благодарит оба института за теплое гостеприимство и превосходные условия для работы. 
что $u=\operatorname{cl}_{X \times X}(Z)$ и

$$
u^{*}: H^{*}(X, \mathbb{Q}) \stackrel{\mathrm{pr}_{1}^{*}}{\longrightarrow} H^{*}(X \times X, \mathbb{Q}) \stackrel{\cup u}{\longrightarrow} H^{*}(X \times X, \mathbb{Q}) \stackrel{\mathrm{pr}_{2 *}}{\longrightarrow} H^{*}(X, \mathbb{Q}),
$$

где $\mathrm{pr}_{1}, \mathrm{pr}_{2}-$ канонические проекции.

Пусть $H$ - гладкое гиперплоское сечение $X$. Обозначим через $L$ оператор Лефшеца на $H^{*}(X, \mathbb{Q})$, определенный формулой $L x=\operatorname{cl}_{X}(H) \cup x$. Согласно сильной теореме Лефшеца отображение

$$
L^{d-i}: H^{i}(X, \mathbb{Q}) \rightarrow H^{2 d-i}(X, \mathbb{Q})
$$

является изоморфизмом для любого $i \leqslant d$. Пусть $\Lambda^{d-i}: H^{2 d-i}(X, \mathbb{Q}) \cong H^{i}(X, \mathbb{Q})$ - изоморфизм, обратный к $L^{d-i}$.

СТАНДАРТНАЯ ГИПОТЕЗА ГРОТЕНДИКА $\left(B^{i}(X)[12]\right)$. ДЛя данного натурального числа $i \leqslant d$ изоморфизм $\Lambda^{d-i}: H^{2 d-i}(X, \mathbb{Q}) \rightarrow H^{i}(X, \mathbb{Q})$ является алгебраическим.

Если $B^{i}(X)$ верна для любого натурального числа $i$, то стандартная гипотеза $B(X)$ верна для $X$.

Гипотеза $B(X)$ влечет алгебраичность классических операторов ${ }^{\mathrm{c}} *$ и $^{\mathrm{c}} \Lambda$ теории Ходжа для $X$, совпадение численной и гомологической эквивалентностей алгебраических циклов на $X$ и алгебраичность компонент Кюннета класса алгебраического цикла на $X \times X$.

Свойство гипотезы $B(X)$ не зависит от поляризащии $L$ и стабильно относительно произведения, гладкого гиперплоского сечения и специализации. Оно выполняется для проективного пространства и других обычных рациональных многообразий, допускающих клеточные разбиения, для кривых, поверхностей и абелевых многообразий. В $\S 11$ к этому списку добавлены некоторые комплексные абелевы схемы над гладкими проективными кривыми.

Пусть $k$ - конечно порожденное расширение простого поля, и пусть $X$ - гладкое проективное многообразие над $k$. Обозначим через $k^{\mathrm{sep}}$ максимальное сепарабельное алгебраическое расширение поля $k$.

ГИПОТЕЗА ТЭЙТА (Tate $\left.{ }^{i}(X)[32]\right)$. Для данного натурального числа $i$ существует такое конечное расширение Галуа $k^{\prime}$ поля $k$, что $\mathbb{Q}_{l}$-пространство

$$
\left[H_{\mathrm{et}}^{2 i}\left(X \otimes_{k} k^{\mathrm{sep}}, \mathbb{Q}_{l}(i)\right)\right]^{\operatorname{Gal}\left(k^{\mathrm{sep}} / k^{\prime}\right)}
$$

порождается классами $k^{\prime}$-рациональных алгебраических ииклов коразмерносmu $i$ на $X \otimes_{k} k^{\prime}$.

Гипотеза Тэйта и соответствующая гипотеза о полюсах дзета-функций обсуждаются в [32], [33].

Значение гипотезы $B(X)$ основано на следующих важных замечаниях:

i) по теореме И. Андре [1, теорема 0.6.2] алгебраичность изоморфизма

$$
\Lambda^{d+1-i}: H^{2 d+2-i}(X, \mathbb{Q}) \rightarrow H^{i}(X, \mathbb{Q})
$$


для всех комплексных абелевых схем $\pi: X \rightarrow C$ относительной размерности $d$ над гладкими проективными кривыми $C$ и всех $i$ влечет гипотезу Ходжа для всех абелевых многообразий;

ii) по теореме Польмана [25, теорема 4] гипотеза Ходжа для комплексного абелева многообразия $X$ СМ-типа эквивалентна гипотезам Тэйта [32] об алгебраических циклах на модели $X_{0}$ многообразия $X$ над числовым полем и полюсах дзета-фрунции $X_{0}[25$, теорема 5];

iii) по теореме Милна [22, теорема 7.1] гипотеза Ходжа для всех абелевых многообразий СМ-типа над $\mathbb{C}$ влечет гипотезы Тэйта [32] об алгебраических циклах и полюсах дзета-функций для всех абелевых многообразий над конечными полями.

Пусть $\pi: X \rightarrow C$ - комплексная абелева схема с главной поляризацией над гладкой проективной кривой, $j: X \times_{C} X \hookrightarrow X \times X$ - каноническое вложение, $\tau: X \times{ }_{C} X \rightarrow C$-структурный морфизм, $\Delta: X \hookrightarrow X \times X$ - диагональное вложение.

Обозначим через $\mathscr{P}$ расслоение Пуанкаре на $X \times_{C} \widehat{X} \stackrel{\rightarrow}{\rightarrow} \times_{C} X$, и пусть $v-$ образ $\operatorname{cl}_{X \times_{C} X}(\mathscr{P}) \in H^{2}\left(X \times_{C} X, \mathbb{Q}\right)$ при каноническом краевом морфизме

$$
H^{2}\left(X \times_{C} X, \mathbb{Q}\right) \rightarrow H^{0}\left(C, R^{2} \tau_{*} \mathbb{Q}\right)
$$

спектральной последовательности Лере

$$
E_{2}^{p, q}=H^{p}\left(C, R^{q} \tau_{*} \mathbb{Q}\right) \Rightarrow H^{*}\left(X \times_{C} X, \mathbb{Q}\right) .
$$

Тогда $j_{*} v^{i}=j_{*}\left(v^{\cup} i\right)-$ канонически определенный алгебраический класс, задающий алгебраический изоморфизм

$$
\left[j_{*} v^{i}\right]^{*}: H^{2}\left(C, R^{2 d-i} \pi_{*} \mathbb{Q}\right) \stackrel{\longrightarrow}{\rightarrow} H^{2}\left(C, R^{i} \pi_{*} \mathbb{Q}\right)
$$

Разовьем подход И. Андре и сведем гипотезу Ходжа для абелевых многообразий к вопросу о существовании алгебраического изоморфизма

$$
H^{2}\left(C, R^{2 d-i} \pi_{*} \mathbb{Q}\right) \stackrel{\sim}{\rightarrow} H^{0}\left(C, R^{i} \pi_{*} \mathbb{Q}\right)
$$

для всех $i \geqslant 2$ и для всех комплексных абелевых схем $\pi: X \rightarrow C$ с главной поляризацией относительной размерности $d$ над гладкими проективными кривыми.

В настоящей статье мы доказываем следующий технический результат.

Пусть $\pi: X \rightarrow C$ - комплексная абелева схема с главной поляризачией относительной размерности $d$ над гладкой проективной кривой $C$. Тогда для любого натурального числа $i \geqslant 2$ существует единственньй цикл Ходжса

$$
\alpha_{i}=\alpha_{i}(X / C) \in\left[H^{0}\left(C, R^{i} \pi_{*} \mathbb{Q}\right) \otimes H^{0}\left(C, R^{i} \pi_{*} \mathbb{Q}\right)\right] \cap H^{i, i}(X \times X, \mathbb{C})
$$

характеризуемьй следующим условием: $\alpha_{i} \cup \mathrm{cl}_{X \times X}\left(X \times X_{s}\right)$ определяет изоморфизм

$$
H^{2}\left(C, R^{2 d-i} \pi_{*} \mathbb{Q}\right) \stackrel{\sim}{\rightarrow} H^{2}\left(C, R^{i} \pi_{*} \mathbb{Q}\right),
$$

совпадающий с алгебраическим изоморфизмом

$$
\left[j_{*} v^{i}\right]^{*}: H^{2}\left(C, R^{2 d-i} \pi_{*} \mathbb{Q}\right) \stackrel{\rightarrow}{\rightarrow} H^{2}\left(C, R^{i} \pi_{*} \mathbb{Q}\right) .
$$


Если $\alpha_{i}$ алгебраччен, то алгебраический класс

$$
\left[\Delta_{*} \mathrm{cl}_{X}\left(X_{s}\right)\right] \circ\left[j_{*} v^{i-2}\right]+j_{*} v^{i-1}+\alpha_{i}
$$

индуцирует алгебрачческий изоморфизм $H^{2 d+2-i}(X, \mathbb{Q}) \widetilde{\rightrightarrows} H^{i}(X, \mathbb{Q})$ и гипотеза $B^{i}(X)$ верна (теорема 10.1).

Если канонически определенные циклы Ходжа $\alpha_{i}(X / C) \in H^{0}\left(C, R^{i} \pi_{*} \mathbb{Q}\right) \otimes$ $H^{0}\left(C, R^{i} \pi_{*} \mathbb{Q}\right)$ алгебраичны для всех натуральных чисел $i \geqslant 2$, то стандартная гипотеза Гротендика $B(X)$ об алгебраичности операторов $\Lambda$ и $*$ верна для $X$.

Докажем гипотезу $B(X)$ для абелевой схемы при условии, что группа Ходжа обшего геометрического слоя $X_{0 \bar{\eta}}$ является простой алгебраической группй типа $C_{d}$ (см. теорему 11.2). Это предположение выполняется для абелевой схемы $\pi: X \rightarrow C$, если $\operatorname{End}\left(X_{s}\right)=\mathbb{Z}$ для некоторого геометрического слоя $X_{s}$ и $d$ - неисключительная размерность (например, если $d$ - нечетное число; см. следствия 11.4, 11.5, 11.3).

Автор благодарен Ю. И. Манину за интересные обсуждения проблемы.

\section{$\S 1$. О некоторых гипотезах, касающихся алгебраических циклов}

1.1. Пусть $X$ - гладкое $d$-мерное проективное многообразие над полем комплексных чисел $\mathbb{C}$. Для $j \geqslant 0$ имеем примитивное разложение Лефшеца

$$
H^{j}(X, \mathbb{Q})=\bigoplus_{k \geqslant \max (0, j-d)} L^{k} P^{j-2 k}(X),
$$

где $P^{i}(X)=H^{i}(X, \mathbb{Q}) \cap \operatorname{Ker} L^{d-i+1}, i \leqslant d,-$ примитивная часть $H^{i}(X, \mathbb{Q})$ (см. [4, $\S 3,(3.4)])$. В частности, любой элемент $x \in H^{j}(X, \mathbb{Q})$ можно записать единственным образом в виде

$$
x=\sum_{k \geqslant \max (0, j-d)} L^{k} x_{j-2 k},
$$

где $x_{j-2 k} \in P^{j-2 k}(X)($ см. $[4, \S 3,(3.3)])$.

Пусть ${ }^{\mathrm{c}} \Lambda$ - двойственный оператор для $L$ в классической теории Ходжа, и пусть $c_{*}$ - классический оператор “звездочка” $[11$, гл. $0, \S 6],[4, \S 3]$. Для элемента $\varphi \in P^{p}(X) \otimes_{\mathbb{Q}} \mathbb{C}$ имеем

$$
{ }^{\mathrm{c}} \Lambda L^{r} \varphi=r(d-p-r+1) L^{r-1} \varphi
$$

(см. $[4, \S 3.1])$. В частности,

${ }^{\mathrm{c}} \Lambda x=\sum_{k \geqslant \max (0, j-d)}{ }^{\mathrm{c}} \Lambda L^{k} x_{j-2 k}=\sum_{k \geqslant \max (0, j-d)} k(d-(j-2 k)-k+1) L^{k-1} x_{j-2 k}$.

Следовательно,

$$
{ }^{\mathrm{c}} \Lambda x=\sum_{k \geqslant \max (1, j-d)} k(d-j+k+1) L^{k-1} x_{j-2 k} .
$$


На пространстве форм типа $(a, b)$ определим оператор Вейля $C$ следуюшей формулой: $C \varphi=(\sqrt{-1})^{a-b} \varphi$. Если $\varphi \in P^{p}(X) \otimes_{\mathbb{Q}} \mathbb{C}$, то

$$
{ }^{c} * L^{r} \varphi= \begin{cases}(-1)^{p(p+1) / 2} \frac{r !}{(d-p-r) !} L^{d-p-r} C \varphi & \text { для } r \leqslant d-p, \\ 0 & \text { для } r>d-p\end{cases}
$$

(см. $[4, \S 3,(3.6)])$. В частности,

$$
\begin{aligned}
{ }^{\mathrm{c}_{* x}} & =\sum_{k \geqslant \max (0, j-d)}{ }^{\mathrm{c}_{*} * L^{k} x_{j-2 k}} \\
& =\sum_{k \geqslant \max (0, j-d)}(-1)^{(j-2 k)(j-2 k+1) / 2} \frac{k !}{(d-(j-2 k)-k) !} L^{d-(j-2 k)-k} C x_{j-2 k} .
\end{aligned}
$$

Поэтому

$$
{ }^{\mathrm{c}_{*}}=\sum_{k \geqslant \max (0, j-d)}(-1)^{(j-2 k)(j-2 k+1) / 2} \frac{k !}{(d-j+k) !} L^{d-j+k} C x_{j-2 k} .
$$

Разложения (1.1), (1.2) позволяют определить следующие абстрактные операторы:

$$
\begin{gathered}
\Lambda x=\sum_{k \geqslant \max (1, j-d)} L^{k-1} x_{j-2 k}, \\
* x=\sum_{k \geqslant \max (0, j-d)}(-1)^{(j-2 k)(j-2 k+1) / 2} L^{d-j+k} x_{j-2 k}, \\
p^{k} x=\delta_{j k} x_{m}, \quad m=\max (0, j-d), \quad k=0, \ldots, 2 d .
\end{gathered}
$$

Формула для $p^{k}$ исправляет формулу (1.4.2.4) из [14]. Все эти формулы обсуждаются в $[14, \S 1.4],[15, \S 4]$. Легко проверить, что $*^{2}=1$ и $\Lambda=* L *[14$, предложение 1.4.3].

Для $i \leqslant d$ изоморфизм $\Lambda^{d-i}: H^{2 d-i}(X, \mathbb{Q}) \rightarrow H^{i}(X, \mathbb{Q})$ является обратным к $L^{d-i}$ и $^{\mathrm{c}} \Lambda^{d-i}: L^{d-i} P^{i}(X) \rightarrow P^{i}(X)$ является обратным к произведению скаляра на $L^{d-i}[14$, предложение 1.4.3], поэтому можно определить инволюцию Леффшеца на $H^{*}(X, \mathbb{Q})[1, \S 1.1]$ :

$$
{ }^{*}{ }_{L} x=\left\{\begin{array}{lll}
L^{d-j} x & \text { для } x \in H^{j}(X, \mathbb{Q}), \quad j \leqslant d, \\
\Lambda^{j-d} x & \text { для } x \in H^{j}(X, \mathbb{Q}), \quad j>d .
\end{array}\right.
$$


1.2. Хорошо известно, что стандартная гипотеза Гротендика $B(X)$ : onepamop $\Lambda$ алгебраичен - эквивалентна любой из следующих гипотез:

1) ${ }^{\mathrm{c}} B(X)$ : оператор ${ }^{\mathrm{c}} \Lambda$ алгебраичен;

2) $*(X)$ : оператор * алгебраичен (см. [15, теорема 4-1]);

3) $\nu(X):$ для любого $i \leqslant \operatorname{dim} X-1$ существует алгебраический изоморфизм

$$
\nu^{i}: H^{2 \operatorname{dim} X-i}(X, \mathbb{Q}) \simeq H^{i}(X, \mathbb{Q})
$$

(см. [14, теорема 2.9]).

(Приведем доказательство эквивалентности $B^{i}(X) \Leftrightarrow \nu^{i}(X)$. Рассмотрим алгебраический изоморфизм

$$
u=\nu^{i} L^{d-i}: H^{i}(X, \mathbb{Q}) \rightarrow H^{i}(X, \mathbb{Q}) .
$$

Его характеристический полином $P(t)$ имеет рациональные коэффициенты. По теореме Кэли-Гамильтона $P(u)=0$, следовательно, $u^{-1}$ является линейной комбинацией $1, u, u^{2}, \ldots$ с рациональными коэффициентами, поэтому $u^{-1}$ алгебраичен, следовательно, изоморфизм

$$
\Lambda^{d-i}: H^{2 d-i}(X, \mathbb{Q}) \stackrel{\sim}{\rightarrow} H^{i}(X, \mathbb{Q})
$$

обратный к $L^{d-i}: H^{i}(X, \mathbb{Q}) \stackrel{\rightrightarrows}{\rightarrow} H^{2 d-i}(X, \mathbb{Q})$ и равный $u^{-1} \nu^{i}$, алгебраичен.)

Пусть $p \leqslant d=\operatorname{dim} X$, и пусть $H_{\mathrm{alg}}^{2 p}(X, \mathbb{Q}) \hookrightarrow H^{2 p}(X, \mathbb{Q})$ - $\mathbb{Q}$-векторное пространство классов алгебраических циклов коразмерности $p$ на $X$. Стандартная гипотеза $B(X)$ имеет следующую более слабую форму.

1.3. ГИПОТЕЗА $A(X, L)$. Для любого натурального числа $p \leqslant d / 2$ инбекиия

$$
L^{d-2 p}: H_{\mathrm{alg}}^{2 p}(X, \mathbb{Q}) \rightarrow H_{\mathrm{alg}}^{2 d-2 p}(X, \mathbb{Q})
$$

является изоморфизмом.

Гипотеза $A(X, L)$ эквивалентна любой из следующих гипотез:

1) $H_{\mathrm{alg}}^{*}(X, \mathbb{Q})$ стабильно относительно оператора $*$;

2) $H_{\mathrm{alg}}^{*}(X, \mathbb{Q})$ стабильно относительно оператора $\Lambda$;

3) $H_{\mathrm{alg}}^{*}(X, \mathbb{Q})$ стабильно относительно оператора ${ }^{\mathrm{c}} \Lambda$

(см. [14, предложение 2.1]).

Согласно классическому результату Д. Либермана гипотеза $A(X, L)$ также эквивалентна следующим гипотезам [19, теорема 1]:

4) $H_{\mathrm{alg}}^{*}(X, \mathbb{Q})$ стабильно относительно оператора ${ }^{{ }^{c}} *$;

5) если $x \in H_{\mathrm{alg}}^{2 p}(X, \mathbb{Q}) u$

$$
x=\sum_{k \geqslant \max (0,2 p-d)} L^{k} x_{2 p-2 k},
$$

где $x_{2 p-2 k} \in P^{2 p-2 k}(X)$, mo $x_{2 p-2 k} \in H_{\mathrm{alg}}^{2 p-2 k}(X, \mathbb{Q})$; 
6) $\mathrm{D}(\mathrm{X})$ : численная әквивалентность алгебраических ииклов на $X$ совпадает с гомологической эквивалентностью.

Последняя гипотеза не зависит от поляризации $L$, поэтому гипотеза $A(X, L)$ не зависит от поляризации, и мы обозначим ее просто $A(X)$.

Утверждаем, что

$$
A(X) \Rightarrow\left(H_{\mathrm{alg}}^{*}(X, \mathbb{Q}) \text { стабильно относительно инволюции Лефшеца } *_{L}\right) .
$$

Действительно, предположим, что гипотеза $A(X)$ верна. Если $x \in H_{\mathrm{alg}}^{2 p}(X, \mathbb{Q})$ и

$$
x=\sum_{k \geqslant \max (0,2 p-d)} L^{k} x_{2 p-2 k}
$$

где $x_{2 p-2 k} \in P^{2 p-2 k}(X)$, то $x_{2 p-2 k} \in H_{\mathrm{alg}}^{2 p-2 k}(X, \mathbb{Q})$. Поскольку $H_{\mathrm{alg}}^{*}(X, \mathbb{Q})$ стабильно относительно операторов $\Lambda$ и $L$, то видно, что $*_{L} x \in H_{\mathrm{alg}}^{*}(X, \mathbb{Q})$.

Напомним, что

$$
B(X) \Rightarrow A(X)
$$

(см. [14, следствие 2.2]) и

$$
A(X \times X) \Rightarrow B(X)
$$

(см. [15, теорема 4-1]),

$$
B(X) \& B(Y) \Rightarrow B(X \times Y)
$$

(см. [14, следствие 2.5]).

Более того,

$$
B(X) \Leftrightarrow A(X \times X)
$$

Действительно, (1.10) влечет соотношение $B(X) \Rightarrow B(X \times X)$; остается заметить, что в силу (1.8), (1.9) имеем $B(X \times X) \Rightarrow A(X \times X)$ и $A(X \times X) \Rightarrow B(X)$.

1.4. Напомним, что $B(X) \Rightarrow C(X)$, где $C(X)$ : компоненты Кюннета $\pi^{i} \otimes$ $H^{2 \operatorname{dim} X-i}(X, \mathbb{Q}) \in H^{i}(X, \mathbb{Q})$ класса диагонали $\Delta$, соответствующие проекииям $\pi^{i}: H^{*}(X, \mathbb{Q}) \rightarrow H^{i}(X, \mathbb{Q})$, алгебраичньь (см. [14, лемма 2.4]).

$\mathrm{C}$ другой стороны, гипотеза $C(X)$ эквивалентна следующему утверждению: $H_{\mathrm{alg}}^{*}(X \times X, \mathbb{Q})$ стабильно относительно разложения Кюннета (см. [14, предложение 2.6]).

1.5. ТЕОРемА. Пусть $X, Y$ - гладкие проективные многообразия над полем комплексных чисел. Если существует сюрвективный морфизм $f: X \rightarrow$ $Y, \operatorname{mo} A(X) \Rightarrow A(Y)$.

ДокАЗАТельство. Напомним, что гипотеза $A(X)$ эквивалентна следующему утверждению: численная и гомологическая эквивалентности совпадают на множестве алгебраических циклов на $X[19$, теорема 1$]$.

Предположим, что $\operatorname{dim} X>\operatorname{dim} Y$. Пусть $X^{\prime}-$ гладкое гиперплоское сечение $X$. Тогда $A(X) \Rightarrow A\left(X^{\prime}\right)$. Действительно, пусть $i: X^{\prime} \hookrightarrow X$-морфизм вложения 
гладкого гиперплоского сечения; тогда $\Lambda^{\prime}=i^{*} \Lambda^{2} i_{*}[14$, предложение $1.4 .7,($ vii)], и поэтому

$$
\begin{aligned}
\Lambda^{\prime}\left(H_{\mathrm{alg}}^{*}\left(X^{\prime}, \mathbb{Q}\right)\right)= & i^{*} \Lambda^{2} i_{*}\left(H_{\mathrm{alg}}^{*}\left(X^{\prime}, \mathbb{Q}\right)\right) \hookrightarrow \\
& \hookrightarrow i^{*} \Lambda^{2}\left(H_{\mathrm{alg}}^{*}(X, \mathbb{Q})\right) \hookrightarrow i^{*}\left(H_{\mathrm{alg}}^{*}(X, \mathbb{Q})\right) \hookrightarrow H_{\mathrm{alg}}^{*}\left(X^{\prime}, \mathbb{Q}\right),
\end{aligned}
$$

так как $i_{*}, \Lambda, i^{*}$ преобразуют алгебраические классы в алгебраические классы. Поскольку $\Lambda^{\prime}$ преобразует алгебраические классы в алгебраические, верна гипотеза $A\left(X^{\prime}\right)$.

С другой стороны, $f_{\left.\right|_{X^{\prime}}}: X^{\prime} \rightarrow Y$ - сюръективный морфизм. Поэтому можно использовать индукцию и свести проблему к случаю $\operatorname{dim} X=\operatorname{dim} Y . \mathrm{B}$ последнем случае пусть $Z_{Y}$ - алгебраический цикл коразмерности $r$ на $Y$, численно эквивалентный нулю на $Y$. Тогда для любого алгебраического цикла $Z_{X}$ коразмерности $d-r$ на $X$ формула проекции влечет следуюшее соотношение:

$$
\operatorname{deg}\left(f_{*}\left(f^{*}\left(\operatorname{cl}_{Y}\left(Z_{Y}\right)\right) \cup \operatorname{cl}_{X}\left(Z_{X}\right)\right)\right)=\operatorname{deg}\left(\operatorname{cl}_{Y}\left(Z_{Y}\right) \cup f_{*}\left(\operatorname{cl}_{X}\left(Z_{X}\right)\right)\right)=0 .
$$

$\mathrm{C}$ другой стороны, $f^{*}\left(\operatorname{cl}_{Y}\left(Z_{Y}\right)\right) \cup \operatorname{cl}_{X}\left(Z_{X}\right) \in H^{2 d}(X, \mathbb{Q}) \simeq \mathbb{Q}$, и поэтому $f_{*}: H^{2 d}(X, \mathbb{Q}) \rightarrow H^{2 d}(Y, \mathbb{Q})$ является изоморфизмом. Следовательно, соотношение (1.12) влечет равенство $f^{*}\left(\operatorname{cl}_{Y}\left(Z_{Y}\right)\right) \cup \mathrm{cl}_{X}\left(Z_{X}\right)=0$ для всех алгебраических циклов $Z_{X}$ коразмерности $d-r$ на $X$. В частности, $f^{*}\left(\operatorname{cl}_{Y}\left(Z_{Y}\right)\right)$ численно эквивалентен нулю. Поскольку гипотеза $A(X)$ верна, то получаем равенство $f^{*}\left(\mathrm{cl}_{Y}\left(Z_{Y}\right)\right)=0$. С другой стороны, формула проекции показывает, что

$$
f_{*} f^{*}\left(\operatorname{cl}_{Y}\left(Z_{Y}\right)\right)=\operatorname{deg} f \cdot \operatorname{cl}_{Y}\left(Z_{Y}\right)
$$

(см. $[9, \S 2.3])$. Следовательно, $\operatorname{cl}_{Y}\left(Z_{Y}\right)=0$ и $Z_{Y}$ гомологически эквивалентен нулю.

Приведем другое доказательство: поскольку $f^{*}$ инъективно $[14$, предложение 1.2.4], то имеем соотношение

$$
f^{*}\left(\operatorname{cl}_{Y}\left(Z_{Y}\right)\right)=0 \Rightarrow \operatorname{cl}_{Y}\left(Z_{Y}\right)=0 \text {. }
$$

Поэтому численная и гомологическая эквивалентности алгебраических циклов совпадают на $Y$ и гипотеза $A(Y)$ верна. Теорема доказана.

1.6. ТЕОремА. Пусть $X, Y$ - гладкие проективные многообразия над полем комплексных чисел. Если существует сюрдективный морфизм $f: X \rightarrow$ $Y, \operatorname{mo} B(X) \Rightarrow B(Y)$.

ДокаЗАТЕЛЬСтво. Поскольку $f \times f: X \times X \rightarrow Y \times Y$ - сюръективный морфизм, теорема из п. 1.5 влечет соотношение $A(X \times X) \Rightarrow A(Y \times Y)$, и утверждение следует из соотношения (1.11). Теорема доказана.

1.7. СлЕДСТВИЕ. Пусть $\operatorname{Sym}^{n}(C)$ - симметрическая степень гладкой проективной кривой $C$ над полем комплексньх чисел. Тогда верна гипотеза $B\left(\operatorname{Sym}^{n}(C)\right)$.

Действительно, поскольку $B(X)$ тривиально выполняется для кривых, остается применить (1.10) и теорему из п. 1.6 к естественному сюръективному морфизму $C^{n}=C \times \cdots \times C \rightarrow \operatorname{Sym}^{n}(C)$. 
1.8. СлЕДСтвиЕ (теорема Д. Либермана [19, теорема 3], [14, теорема 2A11]). Гипотеза $B(X)$ верна для всех абелевых многообразий.

ДокАЗАТЕЛЬСТво. Для любой гладкой проективной кривой $C$ рода $g$ существует морфизм $\operatorname{Sym}^{g}(C) \rightarrow \operatorname{Pic}^{0}(C)$ конечной степени, определенный следуюшей формулой:

$$
f\left(c_{1}+c_{2}+\cdots+c_{g}\right)=\mathscr{O}_{C}\left(c_{1}+c_{2}+\cdots+c_{g}-g \cdot e\right) \in \operatorname{Pic}^{0}(C),
$$

где $e$ - фиксированная точка $C$ [11, гл. II, $\S 7],[26$, гл. V, $\S 1$, предложение 2]. В силу следствия из п. 1.7 и теоремы из п. 1.6 гипотеза $B(X)$ верна для всех якобиевых многообразий. С другой стороны, любое абелево многообразие изоморфно фактору произведения якобиевых многообразий [26, гл. VII, $\S 2.13$, следствие]. Поскольку гипотеза $B(X)$ стабильна относительно произведений (свойство (1.10)), остается применить теорему из п. 1.6.

1.9. Пусть $X$ - гладкое проективное $d$-мерное многообразие над полем $\mathbb{C}$ комплексных чисел. Напомним, что существует канонический изоморфизм ориентации

$$
\operatorname{tr}: H^{2 d}(X, \mathbb{Q}) \stackrel{\rightarrow}{\rightarrow},
$$

и по теореме двойственности Пуанкаре [14, 1.2.А] билинейная форма

$$
\operatorname{tr}(x \cup y): H^{i}(X, \mathbb{Q}) \times H^{2 d-i}(X, \mathbb{Q}) \rightarrow \mathbb{Q}
$$

является совершенной двойственностью (которая отождествляет $H^{i}(X, \mathbb{Q})$ с двойственным пространством к $\left.H^{2 d-i}(X, \mathbb{Q})\right)$.

\section{§ 2. О теореме И. Андре}

2.1. Теорема (Андре [1, теорема 0.6.2]). Любой иикл Ходжа многообразии $J$ является мотивированным (тотіvе́). Более точно, $\xi$ является суммой ииклов вида $\operatorname{pr}_{J *}\left(\alpha \cup{ }_{*} \beta\right)$, где $\alpha$ и $\beta$ - алгебраические чикльи (c коэффичиентами из $\mathbb{Q})$ на $J \times I \times Y_{1} \times \cdots \times Y_{n}, I$ - абелево многообразие, $Y_{i}$ - тотальное пространство компактного пучка абелевых многообразий $u$ $\mathrm{pr}_{J}-$ каноническая проекиия на J.

Напомним, что инволюция Лефшеца $*_{L}$ определена формулой (1.6). Если гипотеза $A\left(J \times I \times Y_{1} \times \cdots \times Y_{n}\right)$ верна, то в силу $(1.7) *_{L}$ преобразует алгебраические классы в алгебраические и (в силу теоремы Андре) гипотеза Ходжа верна для абелева многообразия $J$. С другой стороны, в силу соотношения (1.8)

$$
B\left(J \times I \times Y_{1} \times \cdots \times Y_{n}\right) \Rightarrow A\left(J \times I \times Y_{1} \times \cdots \times Y_{n}\right) .
$$


2.2. По свойству (1.10) гипотеза $B(X)$ стабильна относительно произведения; другими словами, гипотезы $B(X), B(Y)$ влекут $B(X \times Y)$. Следовательно, гипотеза Ходжа верна для абелева многообразия $J$, если стандартная гипотеза $B(X)$ выполняется для всех абелевых многообразий и для всех тотальных пространств компактных пучков абелевых многообразий. По классической теореме Д. Либермана (следствие из п. 1.8) гипотеза $B(X)$ верна для всех абелевых многообразий. Остается доказать $B(X)$ для тотального пространства компактного пучка $\pi: X \rightarrow C$ абелевых многообразий.

Заметим, что для некоторого разветвленного накрытия $C^{\prime} \rightarrow C$ морфизм $X \times{ }_{C}$ $C^{\prime} \rightarrow C^{\prime}$ имеет сечение (другими словами, $X \times{ }_{C} C^{\prime} \rightarrow C^{\prime}$ - абелева схема над $C^{\prime}$ ). Канонический морфизм $X \times_{C} C^{\prime} \rightarrow X$ конечен, и в силу теоремы из п. 1.6 можно полагать, что $\pi: X \rightarrow C$ - абелева схема.

Следовательно, приходим к следуюшему результату [1, с. 33, замечание 2].

2.3. СлЕДСтвИЕ. Если стандартная гипотеза Гротендика $B(X)$ верна для всех абелевых схем $\pi: X \rightarrow C$ над гладкими проективными кривыми $C$, то гипотеза Ходжа верна для всех абелевьх многообразий.

2.4. Пусть $K$ - функциональное поле с полем констант $k$, и пусть $A$ - абелево многообразие над $K$. По определению $K / k$-след $A$ - это пара $(B, \tau)$, состоящая из абелева многообразия $B$ над $k$ и $K$-гомоморфизма $\tau: B \rightarrow A$, обладающего следующим универсальным свойством: для любого абелева многообразия $J$ над $k$ и для любого $K$-гомоморфизма $\varphi: J \rightarrow A$ существует такой единственньй $k$-гомоморфизм $\varphi_{*}: J \rightarrow B$, что диаграмма

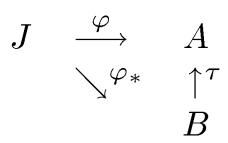

коммутативна.

Хорошо известно, что $K / k$-след сушествует [16, гл. VIII, $\S 3$, теорема 8] и $\tau$ инъективен. Напомним теорему Ленга-Нерона.

2.5. ТЕОрема $[17$, гл. 6 , теорема 2]. Пусть $K$ - функииональное поле с полем констант $k, A$ - абелево многообразие над $K$ и пара $(B, \tau)$ является $K / k$-следом $A$. Тогда группа $A(K) / \tau B(k)$ конечно порождена.

2.6. ЛЕммА. Пусть $\pi: X \rightarrow C$ - абелева схема над гладкой проективной кривой $C, \quad J \times C \stackrel{\mathrm{pr}_{2}}{\longrightarrow} C-$ максимальная постоянная абелева подсхема схемы $\pi: X \rightarrow C$, и пусть $\pi^{\prime}: Y \rightarrow C$ - соответствующая абелева факторсхема. Тогда

$$
\begin{gathered}
B(X) \Leftrightarrow B(Y), \\
H^{0}\left(C, R^{1} \pi_{*}^{\prime} \mathbb{Q}\right)=0 .
\end{gathered}
$$


ДоКАЗАТЕЛЬСТВО. По теореме из п. 1.6 выполнено $B(X) \Rightarrow B(Y)$, Потому что сушествует канонический сюръективный морфизм $X \rightarrow Y$. С другой стороны, теорема Пуанкаре о полной приводимости показывает, что существует изогения абелевых $C$-схем

$$
Y \times J=Y \times{ }_{C}(J \times C) \rightarrow X .
$$

По теореме из п. 1.6 имеем $B(Y \times J) \Rightarrow B(X)$. В силу свойства (1.10) выполнено $B(Y) \& B(J) \Rightarrow B(Y \times J)$. Поскольку гипотеза $B(J)$ верна для всех абелевых многообразий (по теореме Либермана), то получаем соотношение $B(Y) \Rightarrow B(X)$.

Пусть $K=\mathbb{C}(C)$ - поле рациональных функций на кривой $C$. В рассматриваемой ситуации обший слой $Y_{\eta}$ морфизма $\pi^{\prime}: Y \rightarrow C$ имеет тривиальньй $K / \mathbb{C}$-след. По теореме Ленга-Нерона из п. $2.5 Y_{\eta}(K)$ - конечно порожденная группа.

Каноническое экспоненциальное отображение определяет точную последовательность пучков сечений

$$
0 \rightarrow T_{\mathbb{Z}}(Y) \rightarrow\left[e^{*} \Omega_{Y / C}^{1}\right]^{\wedge} \rightarrow Y \rightarrow 0
$$

где $e: C \rightarrow Y$ - нулевое сечение схемы $\pi^{\prime}: Y \rightarrow C$ и

$$
T_{\mathbb{Z}}(Y)=\left[R^{1} \pi_{*}^{\prime} \mathbb{Z}\right]^{\wedge}
$$

- локальная система, двойственная к $R^{1} \pi_{*}^{\prime} \mathbb{Z}$. Мы приходим к точной последовательности когомологий

$$
0 \rightarrow H^{0}\left(C, T_{\mathbb{Z}}(Y)\right) \rightarrow H^{0}\left(C,\left[e^{*} \Omega_{Y / C}^{1}\right]^{\wedge}\right) \rightarrow H^{0}(C, Y) .
$$

В силу [2, лемма 1 в добавлении к $\S 2]$ множество всех продолжений $e: C \rightarrow Y$ до $C$-морфизмов $C[\varepsilon] /\left(\varepsilon^{2}\right) \rightarrow Y$ изоморфно $\operatorname{Hom}_{\mathscr{O}_{C}}\left(e^{*} \Omega_{Y / C}^{1}, \mathscr{O}_{C}\right)$. Поэтому

$$
\operatorname{Hom}_{\mathscr{O}_{C}}\left(e^{*} \Omega_{Y / C}^{1}, \mathscr{O}_{C}\right)=H^{0}\left(C,\left[e^{*} \Omega_{Y / C}^{1}\right]^{\wedge}\right)
$$

- касательное пространство в нуле $\mathbb{C}$-схемы $\operatorname{Sect}_{Y / C}$ сечений $Y$ над $C$ и $\operatorname{Sect}_{Y / C}(\mathbb{C})=Y_{\eta}(K)$. В силу теоремы Ленга-Нерона $\operatorname{dim} H^{0}\left(C,\left[e^{*} \Omega_{Y / C}^{1}\right]^{\wedge}\right)$ равна размерности $K / \mathbb{C}$-следа $Y_{\eta}$. Поэтому

$$
H^{0}\left(C,\left[e^{*} \Omega_{Y / C}^{1}\right]^{\wedge}\right)=0
$$

и из точной последовательности (2.1) получаем

$$
H^{0}\left(C, T_{\mathbb{Z}}(Y)\right)=0
$$

Поскольку $T_{\mathbb{Z}}(Y) \otimes \mathbb{Q}$ - поляризуемая система $\mathbb{Q}$-структур Ходжа веса -1 , существует изоморфизм систем $\mathbb{Q}$-структур Ходжа

$$
R^{1} \pi_{*}^{\prime} \mathbb{Q}=\left[T_{\mathbb{Z}}(Y) \otimes \mathbb{Q}\right]^{\wedge} \simeq T_{\mathbb{Z}}(Y) \otimes \mathbb{Q}(-1)
$$

(см. [6, § 4.2.3]). Поэтому (2.2) влечет соотношения

$$
H^{0}\left(C, R^{1} \pi_{*}^{\prime} \mathbb{Q}\right)=H^{0}\left(C, T_{\mathbb{Z}}(Y) \otimes \mathbb{Q}(-1)\right)=H^{0}\left(C, T_{\mathbb{Z}}(Y)\right) \otimes \mathbb{Q}(-1)=0 .
$$

Лемма доказана.

7

Серия математическая, №3 
2.7. ЗАмечАниЕ. Можно считать, что абелева схема $\pi: X \rightarrow C$ удовлетворяет следуюшему дополнительному условию: для любого (разветвленного) накрытия $C^{\prime} \rightarrow C$ абелева схема $X \times_{C} C^{\prime} \rightarrow C^{\prime}$ не содержит нетривиальных постоянных абелевых подсхем; в частности,

$$
H^{0}\left(C, R^{1} \pi_{*} \mathbb{Q}\right)=0 .
$$

Хорошо известно, что для абелевой схемы $\pi: X \rightarrow C$ сушествует такая изогения $X^{\prime} \rightarrow X$ над $C$, что абелева схема $X^{\prime} \rightarrow C$ имеет главную поляризацию. По теореме из п. 1.6 выполнено $B\left(X^{\prime}\right) \Rightarrow B(X)$. Поэтому можно также считать, что $\pi: X \rightarrow C$ имеет главную поляризацию.

\section{§ 3. О когомологиях комплексных абелевых схем над гладкими проективными кривыми}

3.1. Пусть $C$ - гладкая проективная кривая над полем комплексных чисел и $\pi: X \rightarrow C$ - абелева схема относительной размерности $d$. Поскольку $\pi$ - гладкий морфизм компактных кэлеровых многообразий, то спектральная последовательность Лере $E_{2}^{p, q}=H^{p}\left(C, R^{q} \pi_{*} \mathbb{Q}\right) \Rightarrow H^{*}(X, \mathbb{Q})$ вырождается в члене $E_{2}$, и поэтому

$$
H^{i}(X, \mathbb{Q})=\bigoplus_{a+b=i} H^{a}\left(C, R^{b} \pi_{*} \mathbb{Q}\right)
$$

(см. [5], $[11, \S 3.5])$.

Пусть $\Theta_{n}$ - эндоморфизм $X$, индуцированньй умножением на $n$ в слоях $\pi: X \rightarrow$ $C$, для любого целого числа $n>1$. Поскольку $\left[\Theta_{n}\right]^{*}$ коммутирует с $d_{2}$ дифференциалами спектральной последовательности Лере $E_{2}^{p, q}=H^{p}\left(C, R^{q} \pi_{*} \mathbb{Q}\right) \Rightarrow$ $H^{*}(X, \mathbb{Q})$, то видно, что $H^{a}\left(C, R^{b} \pi_{*} \mathbb{Q}\right)$ совпадает с собственным подпространством $H^{\langle a, b\rangle}(X, \mathbb{Q})$ оператора $\left[\Theta_{n}\right]^{*}$ в $H^{r}(X, \mathbb{Q})$, отвечающим собственному числу $n^{b}[14$, лемма $2 \mathrm{~A} 3]$. Поэтому получаем хорошо известное разложение $\mathbb{Q}$-структур Ходжа

$$
H^{i}(X, \mathbb{Q})=\bigoplus_{a+b=i} H^{\langle a, b\rangle}(X, \mathbb{Q})=\bigoplus_{a+b=i} H^{a}\left(C, R^{b} \pi_{*} \mathbb{Q}\right)
$$

Более того, по той же причине $\cup$-произведение в $H^{*}(X, \mathbb{Q})$ имеет следующее свойство:

$$
H^{a}\left(C, R^{b} \pi_{*} \mathbb{Q}\right) \cup H^{p}\left(C, R^{q} \pi_{*} \mathbb{Q}\right) \hookrightarrow H^{a+p}\left(C, R^{b+q} \pi_{*} \mathbb{Q}\right) .
$$

Легко видеть что $H^{a}\left(C, R^{b} \pi_{*} \mathbb{Q}\right)=(0)$ для всех $a \geqslant 3$. Действительно, $l$-когомологическая размерность есть $\operatorname{cd}_{l}\left(C_{\text {et }}\right) \leqslant 2 \operatorname{dim} C=2$ [21, гл. VI, $\S 1$, теорема 1.1], поэтому $H_{\mathrm{et}}^{a}\left(C, R^{b} \pi_{*} \mathbb{Z} / l^{n} \mathbb{Z}\right)=(0)$ для $a \geqslant 3$, и остается использовать теорему сравнения для этальных когомологий и классических когомологий $[21$, гл. III, $\S 3$, теорема 3.12]. Следовательно, получаем следуюший вариант разложения (3.2):

$$
H^{i}(X, \mathbb{Q})=H^{0}\left(C, R^{i} \pi_{*} \mathbb{Q}\right) \oplus H^{1}\left(C, R^{i-1} \pi_{*} \mathbb{Q}\right) \oplus H^{2}\left(C, R^{i-2} \pi_{*} \mathbb{Q}\right) .
$$


3.2. Напомним что $R^{p} \pi_{*} \mathbb{Q}$ полностью определяется представлением монодромии $\pi_{1}(C, s) \rightarrow \mathrm{GL}\left(H^{p}\left(X_{s}, \mathbb{Q}\right)\right)[11$, гл. $3, \S 5]$.

Пусть $\tau: X \times_{C} X \rightarrow C$ - структурньй морфизм. Тогда $R^{p} \tau_{*} \mathbb{Q}$ полностью определяется соответствующим представлением монодромии

$$
\pi_{1}(C, s) \rightarrow \mathrm{GL}\left(H^{p}\left(X_{s} \times X_{s}, \mathbb{Q}\right)\right)=\mathrm{GL}\left(\bigoplus_{a+b=p} H^{a}\left(X_{s}, \mathbb{Q}\right) \otimes H^{b}\left(X_{s}, \mathbb{Q}\right)\right),
$$

где $\pi_{1}(C, s)$ действует на тензорном произведении $H^{a}\left(X_{s}, \mathbb{Q}\right) \otimes H^{b}\left(X_{s}, \mathbb{Q}\right)$ через диагональное вложение $\pi_{1}(C, s) \hookrightarrow \pi_{1}(C, s) \times \pi_{1}(C, s)=\pi_{1}(C \times C, s \times s)[11$, гл. 3 , $\S 5]$ и разложение Кюннета

$$
H^{p}\left(X_{s} \times X_{s}, \mathbb{Q}\right)=\bigoplus_{a+b=p} H^{a}\left(X_{s}, \mathbb{Q}\right) \otimes H^{b}\left(X_{s}, \mathbb{Q}\right)
$$

согласовано с представлениями монодромии. Поэтому имеем разложение Кюннета

$$
R^{p} \tau_{*} \mathbb{Q}=\bigoplus_{a+b=p} R^{a} \pi_{*} \mathbb{Q} \otimes R^{b} \pi_{*} \mathbb{Q} .
$$

Поскольку $\tau: X \times_{C} X \rightarrow C$ - абелева схема над кривой $C$, то имеем разложения

$$
\begin{aligned}
H^{p}\left(X \times_{C} X, \mathbb{Q}\right)= & H^{0}\left(C, R^{p} \tau_{*} \mathbb{Q}\right) \oplus H^{1}\left(C, R^{p-1} \tau_{*} \mathbb{Q}\right) \oplus H^{2}\left(C, R^{p-2} \tau_{*} \mathbb{Q}\right) \\
= & {\left[\bigoplus_{a+b=p} H^{0}\left(C, R^{a} \pi_{*} \mathbb{Q} \otimes R^{b} \pi_{*} \mathbb{Q}\right)\right] \oplus } \\
& \oplus\left[\bigoplus_{a+b=p-1} H^{1}\left(C, R^{a} \pi_{*} \mathbb{Q} \otimes R^{b} \pi_{*} \mathbb{Q}\right)\right] \oplus \\
& \oplus\left[\bigoplus_{a+b=p-2} H^{2}\left(C, R^{a} \pi_{*} \mathbb{Q} \otimes R^{b} \pi_{*} \mathbb{Q}\right)\right] .
\end{aligned}
$$

Аналогично,

$$
\begin{aligned}
H^{p}(X \times X, \mathbb{Q})= & \bigoplus_{a+b=p}\left[H^{a}(X, \mathbb{Q}) \otimes H^{b}(X, \mathbb{Q})\right] \\
= & \bigoplus_{a+b=p}\left[H^{0}\left(C, R^{a} \pi_{*} \mathbb{Q}\right) \oplus H^{1}\left(C, R^{a-1} \pi_{*} \mathbb{Q}\right) \oplus H^{2}\left(C, R^{a-2} \pi_{*} \mathbb{Q}\right)\right] \otimes \\
& \otimes\left[H^{0}\left(C, R^{b} \pi_{*} \mathbb{Q}\right) \oplus H^{1}\left(C, R^{b-1} \pi_{*} \mathbb{Q}\right) \oplus H^{2}\left(C, R^{b-2} \pi_{*} \mathbb{Q}\right)\right] .
\end{aligned}
$$

Обозначим через $u_{p q r t} \in H^{p}\left(C, R^{q} \pi_{*} \mathbb{Q}\right) \otimes H^{r}\left(C, R^{t} \pi_{*} \mathbb{Q}\right)$ соответствующую компоненту $u \in H^{2 i}(X \times X, \mathbb{Q})$ в разложении (3.6). В частности,

$$
u_{0 i 0 i} \in H^{0}\left(C, R^{i} \pi_{*} \mathbb{Q}\right) \otimes H^{0}\left(C, R^{i} \pi_{*} \mathbb{Q}\right) .
$$


3.3. Лемма. Если $u \in H_{\mathrm{alg}}^{2 i}(X \times X, \mathbb{Q})$, mo $u_{0 i 0 i} \in H_{\mathrm{alg}}^{2 i}(X \times X, \mathbb{Q})$.

ДокАЗАТЕльство. Используем модификацию метода Д. Либермана $[14$, доказательство теоремы 2А11]. В силу (3.2) имеем

$$
\begin{aligned}
{\left[\Theta_{n} \times \mathrm{id}_{X}\right]^{*} u_{p q r t} } & =n^{q} u_{p q r t}, \\
{\left[\operatorname{id}_{X} \times \Theta_{n}\right]^{*} u_{p q r t} } & =n^{t} u_{p q r t} .
\end{aligned}
$$

Для простоты предположим, что $i=2$. Тогда

$$
\begin{aligned}
u= & u_{0004}+u_{0013}+u_{0022}+u_{0103}+u_{0112}+u_{0121}+u_{1003}+u_{1012}+u_{1021} \\
& +u_{0202}+u_{0211}+u_{0220}+u_{1102}+u_{1111}+u_{1120}+u_{2002}+u_{2011}+u_{2020} \\
& +u_{0301}+u_{0310}+u_{1201}+u_{1210}+u_{2101}+u_{2110}+u_{0400}+u_{1300}+u_{2200} .
\end{aligned}
$$

Применяя $\left[\Theta_{n} \times \mathrm{id}_{X}\right]^{*}-\left[\mathrm{id}_{X} \times \mathrm{id}_{X}\right]^{*}$ к этому алгебраическому классу, получим соотношение

$$
\left(\left[\Theta_{n} \times \operatorname{id}_{X}\right]^{*}-\left[\operatorname{id}_{X} \times \operatorname{id}_{X}\right]^{*}\right)(u) \in H_{\text {alg }}^{2 i}(X \times X, \mathbb{Q}) .
$$

В силу (3.7) последнее соотношение имеет вид

$$
\begin{aligned}
(n-1) & u_{0103}+(n-1) u_{0112}+(n-1) u_{0121}+\left(n^{2}-1\right) u_{0202} \\
& +\left(n^{2}-1\right) u_{0211}+\left(n^{2}-1\right) u_{0220}+(n-1) u_{1102}+(n-1) u_{1111} \\
& +(n-1) u_{1120}+\left(n^{3}-1\right) u_{0301}+\left(n^{3}-1\right) u_{0310}+\left(n^{2}-1\right) u_{1201} \\
& +\left(n^{2}-1\right) u_{1210}+(n-1) u_{2101}+(n-1) u_{2110}+\left(n^{4}-1\right) u_{0400} \\
& +\left(n^{3}-1\right) u_{1300}+\left(n^{2}-1\right) u_{2200} \in H_{\mathrm{alg}}^{2 i}(X \times X, \mathbb{Q}) .
\end{aligned}
$$

Следовательно,

$$
\begin{aligned}
u_{0103}+ & u_{0112}+u_{0121}+(n+1) u_{0202}+(n+1) u_{0211}+(n+1) u_{0220}+u_{1102} \\
& +u_{1111}+u_{1120}+\left(n^{2}+n+1\right) u_{0301}+\left(n^{2}+n+1\right) u_{0310}+(n+1) u_{1201} \\
& +(n+1) u_{1210}+u_{2101}+u_{2110}+\left(n^{3}+n^{2}+n+1\right) u_{0400} \\
& +\left(n^{2}+n+1\right) u_{1300}+(n+1) u_{2200} \in H_{\text {alg }}^{2 i}(X \times X, \mathbb{Q}) .
\end{aligned}
$$

Применив $\left[\operatorname{id}_{X} \times \Theta_{n}\right]^{*}-\left[\operatorname{id}_{X} \times \operatorname{id}_{X}\right]^{*}$ к последнему алгебраическому классу, получим соотношение

$$
\begin{aligned}
\left(n^{3}-1\right) & u_{0103}+\left(n^{2}-1\right) u_{0112}+(n-1) u_{0121}+(n+1)\left(n^{2}-1\right) u_{0202} \\
& +(n+1)(n-1) u_{0211}+\left(n^{2}-1\right) u_{1102}+(n-1) u_{1111} \\
& +\left(n^{2}+n+1\right)(n-1) u_{0301}+(n+1)(n-1) u_{1201} \\
& +(n-1) u_{2101} \in H_{\text {alg }}^{2 i}(X \times X, \mathbb{Q}) .
\end{aligned}
$$

Следовательно,

$$
\begin{aligned}
\left(n^{2}+\right. & n+1) u_{0103}+(n+1) u_{0112}+u_{0121} \\
& +(n+1)^{2} u_{0202}+(n+1) u_{0211}+(n+1) u_{1102}+u_{1111} \\
& +\left(n^{2}+n+1\right) u_{0301}+(n+1) u_{1201}+u_{2101} \in H_{\text {alg }}^{2 i}(X \times X, \mathbb{Q}) .
\end{aligned}
$$


Применив $\left[\Theta_{n} \times \operatorname{id}_{X}\right]^{*}-\left[\operatorname{id}_{X} \times \operatorname{id}_{X}\right]^{*}$ к этому алгебраическому классу, получим соотношение

$$
\begin{aligned}
\left(n^{2}+\right. & n+1)(n-1) u_{0103}+(n+1)(n-1) u_{0112}+(n-1) u_{0121} \\
& +(n+1)^{2}\left(n^{2}-1\right) u_{0202}+(n+1)\left(n^{2}-1\right) u_{0211} \\
& +(n+1)(n-1) u_{1102}+(n-1) u_{1111}+\left(n^{2}+n+1\right)\left(n^{3}-1\right) u_{0301} \\
& +(n+1)\left(n^{2}-1\right) u_{1201}+(n-1) u_{2101} \in H_{\mathrm{alg}}^{2 i}(X \times X, \mathbb{Q}) .
\end{aligned}
$$

Поэтому

$$
\begin{aligned}
& \left(n^{2}+n+1\right) u_{0103}+(n+1) u_{0112}+u_{0121} \\
& \quad+(n+1)^{3} u_{0202}+(n+1)^{2} u_{0211}+(n+1) u_{1102}+u_{1111} \\
& \quad+\left(n^{2}+n+1\right)^{2} u_{0301}+(n+1)^{2} u_{1201}+u_{2101} \in H_{\mathrm{alg}}^{2 i}(X \times X, \mathbb{Q}) .
\end{aligned}
$$

Вычитая (3.8) из (3.9), получим следующее соотношение:

$$
\begin{gathered}
(n+1)^{2} n u_{0202}+(n+1) n u_{0211}+\left(n^{2}+n+1\right) n(n+1) u_{0301} \\
+(n+1) n u_{1201} \in H_{\text {alg }}^{2 i}(X \times X, \mathbb{Q}) .
\end{gathered}
$$

Следовательно,

$$
(n+1) u_{0202}+u_{0211}+\left(n^{2}+n+1\right) u_{0301}+u_{1201} \in H_{\mathrm{alg}}^{2 i}(X \times X, \mathbb{Q}) .
$$

Применив $\left[\mathrm{id}_{X} \times \Theta_{n}\right]^{*}-\left[\mathrm{id}_{X} \times \mathrm{id}_{X}\right]^{*}$ к последнему алгебраическому классу, получим соотношение

$$
\begin{aligned}
& (n+1)\left(n^{2}-1\right) u_{0202}+(n-1) u_{0211} \\
& +\left(n^{2}+n+1\right)(n-1) u_{0301}+(n-1) u_{1201} \in H_{\text {alg }}^{2 i}(X \times X, \mathbb{Q}) .
\end{aligned}
$$

Поэтому

$$
(n+1)^{2} u_{0202}+u_{0211}+\left(n^{2}+n+1\right) u_{0301}+u_{1201} \in H_{\text {alg }}^{2 i}(X \times X, \mathbb{Q}) .
$$

Вычитая (3.10) из (3.11), приходим к соотношению $(n+1) n u_{0202} \in H_{\mathrm{alg}}^{2 i}(X \times$ $X, \mathbb{Q})$. В частности, $u_{0 i 0 i} \in H_{\mathrm{alg}}^{2 i}(X \times X, \mathbb{Q})$. Лемма доказана.

\section{§ 4. Расслоения Пуанкаре}

4.1. Пусть $\pi: X \rightarrow C$-абелева схема относительной размерности $d$ надгладкой проективной кривой $C$, и пусть $s \in C$. Напомним, что существует расслоение Пуанкаре $\mathscr{P}_{s}$ на $X_{s} \times \widehat{X}_{s}$, однозначно определенное (с точностью до изоморфизма) следуюшими свойствами:

i) $\left.\mathscr{P}_{s}\right|_{X_{s} \times\left\{L_{s}\right\}} \stackrel{\widetilde{\rightarrow}}{\rightarrow} L_{s}$ для любого элемента $L_{s} \in \widehat{X}_{s}$;

ii) $\left.\mathscr{P}_{s}\right|_{\{0\} \times \widehat{X}_{s}}$ тривиально $[18$, гл. $2, \S 5]$. 
Утверждаем, что $\mathrm{cl}_{X_{s} \times \widehat{X}_{s}}\left(\mathscr{P}_{s}\right)$ имеет тип Кюннета $(1,1)$. Действительно, по формуле Кюннета имеем

$$
\begin{aligned}
& \operatorname{cl}_{X_{s} \times X_{s}}\left(\mathscr{P}_{s}\right) \in H^{2}\left(X_{s} \times X_{s}, \mathbb{Q}\right)= {\left[H^{2}\left(X_{s}, \mathbb{Q}\right) \otimes H^{0}\left(X_{s}, \mathbb{Q}\right)\right] } \\
& \oplus\left[H^{0}\left(X_{s}, \mathbb{Q}\right) \otimes H^{2}\left(X_{s}, \mathbb{Q}\right)\right] \\
& \oplus\left[H^{1}\left(X_{s}, \mathbb{Q}\right) \otimes H^{1}\left(X_{s}, \mathbb{Q}\right)\right] .
\end{aligned}
$$

С другой стороны, по свойствам i), ii) имеем

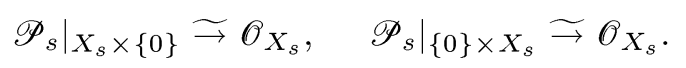

Поэтому $(2,0)$ - и $(0,2)$-компоненты разложения Кюннета $\mathrm{cl}_{X_{s} \times X_{s}}\left(\mathscr{P}_{s}\right)$ тривиальны.

4.2. Пусть $e: C \rightarrow X$ - нулевое сечение и $\hat{\pi}: \widehat{X}=\operatorname{Pic}^{0}(X / C) \rightarrow C$-двойственная абелева схема с нулевым сечением $\hat{e}$. Для любого обратимого пучка $\mathscr{L}$ на $X$ жесткость $\mathscr{L}$ - это изоморфизм $\mathscr{O}_{C} \cong e^{*} \mathscr{L}$. По определению $\operatorname{Pic}^{0}(X / C)$ для любой $C$-схемы $T$ имеем $\operatorname{Hom}_{C}\left(T, \operatorname{Pic}^{0}(X / C)\right)=\{$ классы изоморфизма обратимых пучков $\mathscr{L}$ на $X \times_{C} T$ с такой жесткостью вдоль $e_{T}=e \times_{C} T$, что $\left.\forall t \in T \mathscr{L}\right|_{X \times{ }_{C} t}$ алгебраически эквивалентно нулю на $\left.X \times_{C} t\right\}$ [8, гл. I, $\S 1$, определение 1.4].

Тавтологический обратимый пучок $\mathscr{P}$ на $X \times_{C} \widehat{X}$, отвечаюший тождественному морфизму $\operatorname{id}_{\widehat{X}}: \widehat{X} \rightarrow \widehat{X}=\operatorname{Pic}^{0}(X / C)$, называется расслоением Пуанкаpe. По определению $\mathscr{P}$ обладает жесткостью вдоль $e \times_{C} \widehat{X}$ и $\left.\forall t \in \widehat{X} \mathscr{P}\right|_{X \times_{C} t}$ представляет элемент $\operatorname{Pic}^{0}\left(X \times_{C} t\right)$, задаваемьй точкой $t$ при отождествлении $\operatorname{Pic}^{0}\left(X \times_{C} t\right)=\widehat{X}_{\hat{\pi}(t)}$.

Легко видеть, что $\mathscr{P}$ индуцирует расслоение Пуанкаре $\mathscr{P}_{s}$ на $X_{s} \times \widehat{X}_{s}$ через каноническое вложение $X_{s} \times \widehat{X}_{s} \hookrightarrow X \times_{C} \widehat{X}$. Действительно, имеем каноническое вложение $C$-схем

$$
\widehat{X}_{s} \hookrightarrow \widehat{X} .
$$

Оно определено обратимым пучком $\mathscr{L}=\left.\mathscr{P}\right|_{X \times_{C} \widehat{X}_{s}}$ на $X \times_{C} \widehat{X}_{s}=X_{s} \times \widehat{X}_{s}$, и для любого $t \in \widehat{X}_{s}$

$$
\left.\mathscr{L}\right|_{X_{s} \times_{C} t}=\left.\mathscr{L}\right|_{X_{s} \times t}=\left.\mathscr{P}\right|_{X \times_{C} t}
$$

представляет элемент $\operatorname{Pic}^{0}\left(X_{s} \times t\right)=\operatorname{Pic}^{0}\left(X_{s}\right)$, задаваемый элементом $t$ при отождествлении $\operatorname{Pic}^{0}\left(X_{s}\right)=\widehat{X}_{s}$. Поэтому $\mathscr{L}=\mathscr{P}_{s}$.

4.3. По замечанию из п. 2.7 можно считать, что существует главная поляризация

$$
\lambda: X \stackrel{\longrightarrow}{\longrightarrow}
$$

поэтому можно рассматривать $\lambda$ как отождествление $X=\widehat{X}$. 


\section{$\S 5$. Соответствия и алгебраические изоморфизмы рациональных структур Ходжа}

5.1. Напомним некоторые основные свойства соответствий $[14, \S 1.3]$, [20].

Пусть $X, Y$ - гладкие проективные многообразия над полем комплексных чисел. В силу формулы Кюннета и двойственности Пуанкаре имеются канонические $\mathbb{Q}$-линейные изоморфизмы

$$
\begin{aligned}
H^{*}(X \times Y, \mathbb{Q}) & \widetilde{\rightrightarrows} H^{*}(X, \mathbb{Q}) \otimes_{\mathbb{Q}} H^{*}(Y, \mathbb{Q}) \\
& \widetilde{\rightrightarrows} H_{*}(X, \mathbb{Q}) \otimes_{\mathbb{Q}} H^{*}(Y, \mathbb{Q}) \widetilde{\rightarrow} \operatorname{Hom}_{\mathbb{Q}}\left(H^{*}(X, \mathbb{Q}), H^{*}(Y, \mathbb{Q})\right) .
\end{aligned}
$$

Более точно, элемент $u=a \otimes b \in H^{*}(X \times Y, \mathbb{Q})$ отвечает $\mathbb{Q}$-линейному отображению $u^{*}: H^{*}(X, \mathbb{Q}) \rightarrow H^{*}(Y, \mathbb{Q})$, задаваемому формулой $u^{*}(c)=\operatorname{deg}(c \cup a) \cdot b$, где $\operatorname{deg}: H^{*}(X) \rightarrow \mathbb{Q}$ определяется следуюшей формулой:

$$
\operatorname{deg}=\left\{\begin{array}{l}
\text { изоморфизм ориентации } \operatorname{tr}: H^{2} \operatorname{dim} X(X, \mathbb{Q}) \widetilde{\longrightarrow} \mathbb{Q}, \\
0 \text { на } H^{i}(X, \mathbb{Q}) \text { для } i<2 \operatorname{dim} X .
\end{array}\right.
$$

Произвольный элемент $u \in H^{*}(X \times Y, \mathbb{Q})$ отвечает композиции

$$
u^{*}: H^{*}(X, \mathbb{Q}) \stackrel{\mathrm{pr}_{1}^{*}}{\longrightarrow} H^{*}(X \times Y, \mathbb{Q}) \stackrel{w \rightarrow w \cup u}{\longrightarrow} H^{*}(X \times Y, \mathbb{Q}) \stackrel{\mathrm{pr}_{2 *}}{\longrightarrow} H^{*}(Y, \mathbb{Q}) .
$$

5.2. Линейное отображение $u^{*}: H^{i}(X, \mathbb{Q}) \rightarrow H^{j}(Y, \mathbb{Q})$ называется алгебраическим, если оно является ограничением отображения $u^{*}: H^{*}(X, \mathbb{Q}) \rightarrow H^{*}(Y, \mathbb{Q})$, индуцированного алгебраическим классом $u \in H_{\mathrm{alg}}^{*}(X \times Y, \mathbb{Q})$.

Пусть $V \hookrightarrow H^{i}(X, \mathbb{Q})$ и $W \hookrightarrow H^{j}(Y, \mathbb{Q})$ - $\mathbb{Q}$-структуры Ходжа. Назовем линейное отображение $V \rightarrow W$ алгебраическим, если оно является ограничением отображения $u^{*}: H^{i}(X, \mathbb{Q}) \rightarrow H^{j}(Y, \mathbb{Q})$, индуцированного алгебраическим классом $u \in H_{\text {alg }}^{*}(X \times Y, \mathbb{Q})$.

5.3. Композищия линейных отображений определяет композицию соответствий: если $u \in H^{*}(X \times Y, \mathbb{Q})$ и $v \in H^{*}(Y \times Z, \mathbb{Q})$, то

$$
v \circ u=\operatorname{pr}_{13 *}\left(u \otimes 1_{Z} \cup 1_{X} \otimes v\right) \in H^{*}(X \times Z, \mathbb{Q}),
$$

где $\operatorname{pr}_{13}: X \times Y \times Z \rightarrow X \times Z$ - проекция. Если $X=Y=Z$, то композиция $v \circ u$ алгебраических соответствий является алгебраическим соответствием [14, предложение 1.3.7]. Оно определяется алгебраическим классом

$$
v \circ u=\operatorname{pr}_{13 *}\left(u \otimes 1_{X} \cup 1_{X} \otimes v\right) \in H^{*}(X \times X, \mathbb{Q}) .
$$




\section{§ 6. Основная конструкция}

6.1. Пусть $\mu_{s}: X_{s} \times X_{s} \rightarrow X_{s}-$ групповой закон для $X_{s}$ (морфизм суммирования). Напомним некоторые результаты С. Л. Клеймана и Д. Либермана.

6.2. Лемма [14, лемма $2 \mathrm{~A} 12]$. Пусть $X_{s}$ - абелево многообразие, $Y_{s}$ - произвольное гладкое проективное многообразие $и и \in H^{2}\left(X_{s} \times Y_{s}, \mathbb{Q}\right)$ - әлемент кюннетовского типа $(1,1)$. Тогда соответствие

$$
\exp (u)=\sum u^{i} / i !: H^{*}\left(X_{s}, \mathbb{Q}\right) \rightarrow H^{*}\left(Y_{s}, \mathbb{Q}\right)
$$

преобразует произведение Понтрягина $\alpha \vee \beta=\mu_{s *}(\alpha \otimes \beta)$ в $\cup$-произведение.

6.3. Лемма $[14$, замечание $2 \mathrm{~A} 13]$. Пусть $X_{s}$ - абелево многообразие, $\widehat{X}_{s}-$ двойственное абелево многообразие $и$ u $H^{2}\left(X_{s} \times \widehat{X}_{s}, \mathbb{Q}\right)$ - $(1,1)$-кюннетовская компонента дивизора Пуанкаре. Тогда соответствие

$$
\exp (u)=\sum u^{i} / i !: H^{*}\left(X_{s}, \mathbb{Q}\right) \rightarrow H^{*}\left(\widehat{X}_{s}, \mathbb{Q}\right)
$$

является алгебраическим изоморфизмом алгебры Понтрягина $H^{*}\left(X_{s}, \mathbb{Q}\right)$ с алгеброй, определенной $\cup$-произведением на $H^{*}\left(\widehat{X}_{s}, \mathbb{Q}\right)$.

6.4. В рассматриваемой ситуации $X_{s}=\widehat{X}_{s}$ и $\mathrm{cl}_{X_{s} \times X_{s}}\left(\mathscr{P}_{s}\right)$ имеет тип Кюннета $(1,1)$, поэтому (по лемме из п. 6.3) для любого натурального числа $i$ отображение

$$
H^{2 d-i}\left(X_{s}, \mathbb{Q}\right) \rightarrow H^{i}\left(X_{s}, \mathbb{Q}\right),
$$

определенное классом

$$
\left[\operatorname{cl}_{X_{s} \times X_{s}}\left(\mathscr{P}_{s}\right)\right]^{\cup i}=\operatorname{cl}_{X_{s} \times X_{s}}\left(\mathscr{P}_{s}\right) \cup \cdots \cup \operatorname{cl}_{X_{s} \times X_{s}}\left(\mathscr{P}_{s}\right) \in H^{2 i}\left(X_{s} \times X_{s}, \mathbb{Q}\right),
$$

является алгебраическим изоморфизмом. Более точно, этот изоморфизм является композицией следующих изоморфизмов:

$$
\begin{aligned}
H^{2 d-i}\left(X_{s}, \mathbb{Q}\right) & \stackrel{\mathrm{pr}_{1 s}^{*}}{\longrightarrow} H^{2 d-i}\left(X_{s}, \mathbb{Q}\right) \otimes H^{0}\left(X_{s}, \mathbb{Q}\right)--\cup\left[\mathrm{cl}_{X_{s} \times X_{s}}\left(\mathscr{P}_{s}\right)\right]^{\cup i} \\
& \simeq H^{2 d}\left(X_{s}, \mathbb{Q}\right) \otimes H^{i}\left(X_{s}, \mathbb{Q}\right) \stackrel{\operatorname{pr}_{2 s *}}{\longrightarrow} H^{i}\left(X_{s}, \mathbb{Q}\right),
\end{aligned}
$$

где $\operatorname{pr}_{k s}: X_{s} \times X_{s} \rightarrow X_{s}$-каноническая проекция.

6.5. Пусть $i_{s}: X_{s} \hookrightarrow X$ - каноническое вложение. Тогда мы имеем канонические вложения

$$
X_{s} \times X_{s} \stackrel{i_{s} \times i_{s}}{\hookrightarrow} X \times_{C} X \stackrel{j}{\hookrightarrow} X \times X .
$$

По теореме Делиня [6, 4.1.2] канонический морфизм ограничения

$$
H^{2}\left(X \times_{C} X, \mathbb{Q}\right) \rightarrow H^{2}\left(X_{s} \times X_{s}, \mathbb{Q}\right)
$$


является композицией канонического сюръективного краевого морфизма (рациональных структур Ходжа) $H^{2}\left(X \times_{C} X, \mathbb{Q}\right) \rightarrow H^{0}\left(C, R^{2} \tau_{*} \mathbb{Q}\right)$, ассоциированного со спектральной последовательностью Лере $E_{2}^{p, q}=H^{p}\left(C, R^{q} \tau_{*} \mathbb{Q}\right) \Rightarrow H^{*}\left(X \times_{C}\right.$ $X, \mathbb{Q})$, и канонических отображений

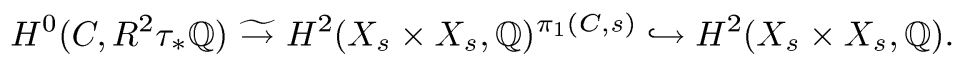

Очевидно, что $\operatorname{cl}_{X \times_{C} X}(\mathscr{P}) \in H^{2}\left(X \times_{C} X, \mathbb{Q}\right)$ отображается в элемент $v_{s}=$ $\mathrm{cl}_{X_{s} \times X_{s}}\left(\mathscr{P}_{s}\right) \in H^{2}\left(X_{s} \times X_{s}, \mathbb{Q}\right)^{\pi_{1}(C, s)}$ типа Кюннета $(1,1)$, так как

$$
\left[i_{s} \times i_{s}\right]^{*} \operatorname{cl}_{X \times_{C} X}(\mathscr{P})=\operatorname{cl}_{X_{s} \times X_{s}}\left(\left[i_{s} \times i_{s}\right]^{*}(\mathscr{P})\right)
$$

$($ см. $[14,1.2 . \mathrm{C}])$ и $\left[i_{s} \times i_{s}\right]^{*}(\mathscr{P})=\mathscr{P}_{s}$. Поэтому

$$
\begin{aligned}
\operatorname{cl}_{X \times_{C} X}(\mathscr{P}) \in H^{0}\left(C, R^{1} \pi_{*} \mathbb{Q} \otimes R^{1} \pi_{*} \mathbb{Q}\right) \oplus \\
\oplus\left[\bigoplus_{a+b=1} H^{1}\left(C, R^{a} \pi_{*} \mathbb{Q} \otimes R^{b} \pi_{*} \mathbb{Q}\right)\right] \oplus H^{2}(C, \mathbb{Q}) .
\end{aligned}
$$

В силу теоремы Лефшеца $H^{0}\left(C, R^{2} \tau_{*} \mathbb{Q}\right)$-компонента $v$ алгебраического класса $\operatorname{cl}_{X \times_{C} X}(\mathscr{P})$ является алгебраической.

$\mathrm{C}$ другой стороны, $v \in H^{0}\left(C, R^{1} \pi_{*} \mathbb{Q} \otimes R^{1} \pi_{*} \mathbb{Q}\right)$.

6.6. Утверждаем, что алгебраический класс

$$
v^{i}=v \cup v \cup \cdots \cup v \in H^{0}\left(C, R^{i} \pi_{*} \mathbb{Q} \otimes R^{i} \pi_{*} \mathbb{Q}\right) \hookrightarrow H^{2 i}\left(X \times_{C} X, \mathbb{Q}\right)
$$

определяет канонический изоморфизм локальных систем

$$
\nu^{i}: R^{2 d-i} \pi_{*} \mathbb{Q} \stackrel{\sim}{\longrightarrow} R^{i} \pi_{*} \mathbb{Q}
$$

над $C$. Действительно, пусть $\nu^{i}-$ композиция

$$
R^{2 d-i} \pi_{*} \mathbb{Q} \stackrel{p_{1}^{*}}{\longrightarrow} R^{2 d-i} \pi_{*} \mathbb{Q} \otimes R^{0} \pi_{*} \mathbb{Q} \stackrel{\cup v^{i}}{\longrightarrow} R^{2 d} \pi_{*} \mathbb{Q} \otimes R^{i} \pi_{*} \mathbb{Q} \stackrel{p_{2 *}}{\longrightarrow} R^{i} \pi_{*} \mathbb{Q},
$$

где $p_{1}, p_{2}-$ канонические проекции $X \times_{C} X \rightarrow X$. В терминах представлений монодромии $\pi_{1}(C, s)$ имеем

$$
v^{i} \in H^{0}\left(C, R^{i} \pi_{*} \mathbb{Q} \otimes R^{i} \pi_{*} \mathbb{Q}\right) \stackrel{\sim}{\rightarrow}\left[H^{i}\left(X_{s}, \mathbb{Q}\right) \otimes H^{i}\left(X_{s}, \mathbb{Q}\right)\right]^{\pi_{1}(C, s)},
$$

и $\nu^{i}-$ композиция изоморфизмов $\pi_{1}(C, s)$-модулей

$$
\begin{aligned}
& H^{2 d-i}\left(X_{s}, \mathbb{Q}\right) \stackrel{p_{1 s}^{*}}{\longrightarrow} H^{2 d-i}\left(X_{s}, \mathbb{Q}\right) \otimes H^{0}\left(X_{s}, \mathbb{Q}\right) \\
& \stackrel{\cup v_{s}^{i}}{\longrightarrow} H^{2 d}\left(X_{s}, \mathbb{Q}\right) \otimes H^{i}\left(X_{s}, \mathbb{Q}\right) \stackrel{p_{2 s *}}{\longrightarrow} H^{i}\left(X_{s}, \mathbb{Q}\right) .
\end{aligned}
$$


Эти последовательности индуцируют следуюшую последовательность изоморфизмов:

$$
\begin{aligned}
H^{k}\left(C, R^{2 d-i} \pi_{*} \mathbb{Q}\right) & \stackrel{p_{1}^{*}}{\longrightarrow} H^{k}\left(C, R^{2 d-i} \pi_{*} \mathbb{Q} \otimes R^{0} \pi_{*} \mathbb{Q}\right) \\
& \stackrel{\cup v^{i}}{\longrightarrow} H^{k}\left(C, R^{2 d} \pi_{*} \mathbb{Q} \otimes R^{i} \pi_{*} \mathbb{Q}\right) \stackrel{p_{2 *}}{\longrightarrow} H^{k}\left(C, R^{i} \pi_{*} \mathbb{Q}\right) .
\end{aligned}
$$

Очевидно, что $p_{k}=\operatorname{pr}_{k} \circ j$, поэтому нашу последовательность можно переписать в виде

$$
\begin{aligned}
H^{k}\left(C, R^{2 d-i} \pi_{*} \mathbb{Q}\right) & \stackrel{j^{*} \operatorname{pr}_{1}^{*}}{\longrightarrow} H^{k}\left(C, R^{2 d-i} \pi_{*} \mathbb{Q} \otimes R^{0} \pi_{*} \mathbb{Q}\right) \\
& \stackrel{\cup v^{i}}{\longrightarrow} H^{k}\left(C, R^{2 d} \pi_{*} \mathbb{Q} \otimes R^{i} \pi_{*} \mathbb{Q}\right) \stackrel{\operatorname{pr}_{2 *} j_{*}}{\longrightarrow} H^{k}\left(C, R^{i} \pi_{*} \mathbb{Q}\right) .
\end{aligned}
$$

Пусть $x \in H^{k}\left(C, R^{2 d-i} \pi_{*} \mathbb{Q}\right)$. Тогда из формулы проекции следует соотношение

$$
\operatorname{pr}_{2 *} j_{*}\left(j^{*} \operatorname{pr}_{1}^{*}(x) \cup v^{i}\right)=\operatorname{pr}_{2 *}\left(\operatorname{pr}_{1}^{*}(x) \cup j_{*} v^{i}\right) .
$$

В частности, алгебраический класс $j_{*} v^{i} \in H^{2 i+2}(X \times X, \mathbb{Q})$ определяет алгебраический изоморфизм

$$
H^{k}\left(C, R^{2 d-i} \pi_{*} \mathbb{Q}\right) \stackrel{\left[j_{*} v^{i}\right]^{*}}{-} \underset{\rightarrow}{\rightarrow} H^{k}\left(C, R^{i} \pi_{*} \mathbb{Q}\right)
$$

6.7. Рассмотрим канонические отображения

$$
\begin{aligned}
& \alpha: H^{1}(\operatorname{Alb}(X), \mathbb{Q}) \rightarrow H^{1}(X, \mathbb{Q}), \\
& \beta: H^{2 d+1}(X, \mathbb{Q}) \rightarrow H^{1}\left(\operatorname{Pic}^{0}(X), \mathbb{Q}\right)
\end{aligned}
$$

из [14, 2A1], где $\beta$ индуцируется дивизором Пуанкаре $D$ на $X \times \operatorname{Pic}^{0}(X)$. Известно, что $\alpha$ - инъекция и $\beta$ - сюръекция [14, теорема 2А9(1)]. Над полем характеристики нуль оба отображения являются изоморфизмами. Более того, существует дивизор $E$ на $X \times X$, который индуцирует скалярное кратное отображение $\Lambda^{d}: H^{2 d+1}(X, \mathbb{Q}) \rightarrow H^{1}(X, \mathbb{Q})$, обратное к $L^{d}[14$, теорема $2 \mathrm{~A} 9(5)]$. В частности, $\Lambda^{d}$ алгебраичен, поэтому гипотеза $B^{1}(X)$ верна.

Другое доказательство гипотезы $B^{1}(X)$ : в силу леммы из п. 2.6 можно считать, что $H^{0}\left(C, R^{1} \pi_{*} \mathbb{Q}\right)=0$; в этом случае

$$
\begin{gathered}
H^{1}(X, \mathbb{Q})=H^{1}(C, \mathbb{Q}), \\
H^{2 d+1}(X, \mathbb{Q})=H^{1}\left(C, R^{2 d} \pi_{*} \mathbb{Q}\right) \oplus H^{2}\left(C, R^{2 d-1} \pi_{*} \mathbb{Q}\right)=H^{1}\left(C, R^{2 d} \pi_{*} \mathbb{Q}\right),
\end{gathered}
$$

потому что $R^{2 d} \pi_{*} \mathbb{Q} \widetilde{\longrightarrow} \mathbb{Q}$. В силу соотношения $(6.2)$ алгебраический класс $j_{*} 1 \in$ $H^{2}(X \times X, \mathbb{Q})$ определяет алгебраический изоморфизм

$$
H^{1}\left(C, R^{2 d} \pi_{*} \mathbb{Q}\right) \stackrel{\Im}{\rightarrow} H^{1}(C, \mathbb{Q}),
$$


и гипотеза $B^{1}(X)$ верна. Очевидно, что для образующей $\gamma 1$-мерного пространства $H^{0}(X, \mathbb{Q})=H_{\mathrm{alg}}^{0}(X, \mathbb{Q})$ каноническая последовательность

$$
\begin{aligned}
H^{2 d+2}(X, \mathbb{Q}) & \stackrel{\mathrm{pr}_{1}^{*}}{\longrightarrow} H^{2 d+2}(X, \mathbb{Q}) \otimes H^{0}(X, \mathbb{Q}) \\
& \stackrel{\cup \gamma}{\longrightarrow} H^{2 d+2}(X, \mathbb{Q}) \otimes H^{0}(X, \mathbb{Q}) \stackrel{\operatorname{pr}_{2 *}}{\longrightarrow} H^{0}(X, \mathbb{Q})
\end{aligned}
$$

определяет алгебраический изоморфизм

$$
H^{2 d+2}(X, \mathbb{Q}) \stackrel{\Im}{\rightarrow} H^{0}(X, \mathbb{Q}) .
$$

В частности, гипотеза $B^{0}(X)$ верна.

Следовательно, можно считать, что $i \geqslant 2$.

В этом случае алгебраические изоморфизмы (6.2) определяют следующую диаграмму:

$$
\begin{array}{ccccc}
H^{0}\left(C, R^{2 d+2-i} \pi_{*} \mathbb{Q}\right) & \oplus & H^{1}\left(C, R^{2 d+1-i} \pi_{*} \mathbb{Q}\right) & \oplus & H^{2}\left(C, R^{2 d-i} \pi_{*} \mathbb{Q}\right) \\
\simeq \downarrow^{\left[j_{*} v^{i-2}\right]^{*}} & & \simeq \downarrow^{\left[j_{*} v^{i-1}\right]^{*}} & & \simeq \downarrow^{\left[j_{*} v^{i}\right]^{*}} \\
H^{0}\left(C, R^{i-2} \pi_{*} \mathbb{Q}\right) & \oplus & H^{1}\left(C, R^{i-1} \pi_{*} \mathbb{Q}\right) & \oplus & H^{2}\left(C, R^{i} \pi_{*} \mathbb{Q}\right)
\end{array}
$$

где все вертикальные стрелки являются алгебраическими изоморфизмами.

Сравнивая строки диаграммы с разложениями

$$
\begin{aligned}
H^{2 d+2-i}(X, \mathbb{Q})= & H^{0}\left(C, R^{2 d+2-i} \pi_{*} \mathbb{Q}\right) \oplus H^{1}\left(C, R^{2 d+1-i} \pi_{*} \mathbb{Q}\right) \oplus \\
& \oplus H^{2}\left(C, R^{2 d-i} \pi_{*} \mathbb{Q}\right), \\
H^{i}(X, \mathbb{Q})= & H^{2}\left(C, R^{i-2} \pi_{*} \mathbb{Q}\right) \oplus H^{1}\left(C, R^{i-1} \pi_{*} \mathbb{Q}\right) \oplus H^{0}\left(C, R^{i} \pi_{*} \mathbb{Q}\right),
\end{aligned}
$$

видим, что морфизм $\left[j_{*} v^{i-1}\right]^{*}$ дает подходящее отображение, но $\left[j_{*} v^{i-2}\right]^{*}$ и $\left[j_{*} v^{i}\right]^{*}$ нуждаются в коррекции, чтобы получить алгебраический изоморфизм $H^{2 d+2-i}(X, \mathbb{Q}) \stackrel{\rightrightarrows}{\rightarrow} H^{i}(X, \mathbb{Q})$.

\section{$\S 7$. Коррекция морфизма $\left[j_{*} v^{i-2}\right]^{*}$}

7.1. Пусть $H$ - гиперплоское сечение $X$, и пусть $h \in H^{0}\left(C, R^{2} \pi_{*} \mathbb{Q}\right)$ - компонента

$$
\operatorname{cl}_{X}(H) \in H^{2}(X, \mathbb{Q})=H^{0}\left(C, R^{2} \pi_{*} \mathbb{Q}\right) \oplus H^{1}\left(C, R^{1} \pi_{*} \mathbb{Q}\right) \oplus H^{2}(C, \mathbb{Q}) .
$$

По теореме Лефшеца видно, что $h$ - алгебраический класс, индуцирующий поляризацию на каждом слое $X_{s}$. Согласно сильной теореме Лефшеца каноническое отображение

$$
H^{i}\left(X_{s}, \mathbb{Q}\right) \stackrel{\cup h^{d-i}}{-}-\stackrel{-\rightarrow}{\rightarrow 2 d-i}\left(X_{s}, \mathbb{Q}\right)
$$

является изоморфизмом $\pi_{1}(C, s)$-модулей $(i \leqslant d)$. Поэтому $h^{d-i}$ определяет изоморфизм

$$
\begin{aligned}
& H^{0}\left(C, R^{i} \pi_{*} \mathbb{Q}\right)=H^{i}\left(X_{s}, \mathbb{Q}\right)^{\pi_{1}(C, s)} \\
& \stackrel{\cup h^{d-i}}{\longrightarrow} H^{2 d-i}\left(X_{s}, \mathbb{Q}\right)^{\pi_{1}(C, s)}=H^{0}\left(C, R^{2 d-i} \pi_{*} \mathbb{Q}\right),
\end{aligned}
$$

и этот изоморфизм индуцируется алгебраическим классом $\Delta_{*} h^{d-i}$, где $\Delta: X \hookrightarrow$ $X \times X$ - диагональное вложение [14, предложение 1.3.5]. Имеем равенство

$$
\operatorname{dim} H^{0}\left(C, R^{i} \pi_{*} \mathbb{Q}\right)=\operatorname{dim} H^{0}\left(C, R^{2 d-i} \pi_{*} \mathbb{Q}\right) .
$$


7.2. Имеется каноническое невырожденное спаривание $\pi_{1}(C, s)$-модулей

$$
H^{2 d-i}\left(X_{s}, \mathbb{Q}\right) \times H^{i}\left(X_{s}, \mathbb{Q}\right) \rightarrow H^{2 d}\left(X_{s}, \mathbb{Q}\right) \stackrel{\operatorname{tr}_{X_{s}}}{\rightarrow} \mathbb{Q} .
$$

Оно влечет отождествление $\pi_{1}(C, s)$-модулей

$$
H^{i}\left(X_{s}, \mathbb{Q}\right)^{\wedge}=H^{2 d-i}\left(X_{s}, \mathbb{Q}\right)
$$

поэтому мы имеем отождествление

$$
\left[H^{i}\left(X_{s}, \mathbb{Q}\right)^{\wedge}\right]^{\pi_{1}(C, s)}=H^{2 d-i}\left(X_{s}, \mathbb{Q}\right)^{\pi_{1}(C, s)}
$$

В частности,

$$
\begin{aligned}
H^{0}\left(C, R^{i} \pi_{*} \mathbb{Q}\right)^{\wedge} & =\left[H^{i}\left(X_{s}, \mathbb{Q}\right)^{\pi_{1}(C, s)}\right]^{\wedge}=\left[H^{i}\left(X_{s}, \mathbb{Q}\right)^{\wedge}\right]^{\pi_{1}(C, s)} \\
& =H^{2 d-i}\left(X_{s}, \mathbb{Q}\right)^{\pi_{1}(C, s)}=H^{0}\left(C, R^{2 d-i} \pi_{*} \mathbb{Q}\right)
\end{aligned}
$$

и каноническое спаривание

$$
H^{0}\left(C, R^{2 d-i} \pi_{*} \mathbb{Q}\right) \times H^{0}\left(C, R^{i} \pi_{*} \mathbb{Q}\right) \rightarrow H^{0}\left(C, R^{2 d} \pi_{*} \mathbb{Q}\right)
$$

невырожденно: для любого элемента $0 \neq y \in H^{0}\left(C, R^{i} \pi_{*} \mathbb{Q}\right)$ существует такой элемент $x \in H^{0}\left(C, R^{2 d-i} \pi_{*} \mathbb{Q}\right)$, что

$$
0 \neq x \cup y \in H^{0}\left(C, R^{2 d} \pi_{*} \mathbb{Q}\right)
$$

Пусть $c$ - образ $\mathrm{cl}_{X}(e(C)) \in H^{2 d}(X, \mathbb{Q})$ относительно канонического морфизма

$$
H^{2 d}(X, \mathbb{Q}) \rightarrow H^{0}\left(C, R^{2 d} \pi_{*} \mathbb{Q}\right)
$$

имеем

$$
\mathrm{cl}_{X}(e(C))=c+c_{1}+c_{2}
$$

где $c_{j} \in H^{j}\left(C, R^{2 d-j} \pi_{*} \mathbb{Q}\right), j=1,2$. Поскольку $\mathrm{cl}_{X}\left(X_{s}\right) \in H^{2}(C, \mathbb{Q})$ - образующая 1-мерного пространства $H^{2}(C, \mathbb{Q})$, то видно, что

$$
\operatorname{cl}_{X}\left(X_{s}\right) \cup c_{j}=0, \quad j=1,2,
$$

и

$$
\operatorname{cl}_{X}\left(X_{s}\right) \cup c=\operatorname{cl}_{X}\left(X_{s}\right) \cup \operatorname{cl}_{X}(e(C))=\operatorname{cl}_{X}\left(X_{s} \cap e(C)\right)=\operatorname{cl}_{X}(e(s)) \neq 0 .
$$

В частности, $0 \neq \operatorname{cl}_{X}\left(X_{s}\right) \cup H^{0}\left(C, R^{2 d} \pi_{*} \mathbb{Q}\right)$. Поскольку последнее пространство содержится в 1 -мерном пространстве $H^{2}\left(C, R^{2 d} \pi_{*} \mathbb{Q}\right)$, то мы получаем равенства

$$
H^{2}\left(C, R^{2 d} \pi_{*} \mathbb{Q}\right)=\operatorname{cl}_{X}\left(X_{s}\right) \cup H^{0}\left(C, R^{2 d} \pi_{*} \mathbb{Q}\right)=H^{2}(C, \mathbb{Q}) \cup H^{0}\left(C, R^{2 d} \pi_{*} \mathbb{Q}\right) .
$$


Следовательно, канонический морфизм

$$
H^{0}\left(C, R^{2 d} \pi_{*} \mathbb{Q}\right) \stackrel{\cup \operatorname{cl}_{X}\left(X_{s}\right)}{-} H^{2}\left(C, R^{2 d} \pi_{*} \mathbb{Q}\right)
$$

биективен и

$$
0 \neq \operatorname{cl}_{X}\left(X_{s}\right) \cup x \cup y \in H^{2}\left(C, R^{2 d} \pi_{*} \mathbb{Q}\right) .
$$

В частности,

$$
0 \neq \operatorname{cl}_{X}\left(X_{s}\right) \cup y \in H^{2}\left(C, R^{i} \pi_{*} \mathbb{Q}\right)
$$

для любого $0 \neq y \in H^{0}\left(C, R^{i} \pi_{*} \mathbb{Q}\right)$; поэтому канонический морфизм

$$
H^{0}\left(C, R^{i} \pi_{*} \mathbb{Q}\right) \stackrel{\cup \mathrm{cl}_{X}\left(X_{s}\right)}{\longrightarrow} H^{2}\left(C, R^{i} \pi_{*} \mathbb{Q}\right)
$$

инъективен.

Заметим, что элементы $H^{2}\left(C, R^{i} \pi_{*} \mathbb{Q}\right)$ аннулируют ( $\cup$-произведением) компоненты $H^{1}\left(C, R^{2 d-i-1} \pi_{*} \mathbb{Q}\right)$ и $H^{2}\left(C, R^{2 d-i-2} \pi_{*} \mathbb{Q}\right)$ пространства

$$
H^{2 d-i}(X, \mathbb{Q})=H^{0}\left(C, R^{2 d-i} \pi_{*} \mathbb{Q}\right) \oplus H^{1}\left(C, R^{2 d-i-1} \pi_{*} \mathbb{Q}\right) \oplus H^{2}\left(C, R^{2 d-i-2} \pi_{*} \mathbb{Q}\right)
$$

поэтому невырожденность канонического спаривания

$$
H^{i+2}(X, \mathbb{Q}) \times H^{2 d-i}(X, \mathbb{Q}) \rightarrow H^{2 d+2}(X, \mathbb{Q}) \stackrel{\text { tr }}{\rightarrow} \mathbb{Q}
$$

(теорема двойственности Пуанкаре) влечет невырожденность канонического спаривания

$$
H^{2}\left(C, R^{i} \pi_{*} \mathbb{Q}\right) \times H^{0}\left(C, R^{2 d-i} \pi_{*} \mathbb{Q}\right) \rightarrow H^{2}\left(C, R^{2 d} \pi_{*} \mathbb{Q}\right) \stackrel{\text { tr }}{\rightarrow} \mathbb{Q} .
$$

В частности,

$$
H^{2}\left(C, R^{i} \pi_{*} \mathbb{Q}\right)^{\wedge} \underset{\rightarrow}{\longrightarrow} H^{0}\left(C, R^{2 d-i} \pi_{*} \mathbb{Q}\right)
$$

Поэтому в силу равенства (7.1)

$$
\begin{aligned}
\operatorname{dim} H^{2}\left(C, R^{i} \pi_{*} \mathbb{Q}\right) & =\operatorname{dim} H^{2}\left(C, R^{i} \pi_{*} \mathbb{Q}\right)^{\wedge}=\operatorname{dim} H^{0}\left(C, R^{2 d-i} \pi_{*} \mathbb{Q}\right) \\
& =\operatorname{dim} H^{0}\left(C, R^{i} \pi_{*} \mathbb{Q}\right)
\end{aligned}
$$

и инъективный морфизм (7.2) биективен:

$$
H^{0}\left(C, R^{i} \pi_{*} \mathbb{Q}\right) \stackrel{\cup l_{X}\left(X_{s}\right)}{-} \stackrel{-}{\longrightarrow} H^{2}\left(C, R^{i} \pi_{*} \mathbb{Q}\right)
$$


7.3. В рассматриваемой ситуации

$$
H^{2}\left(C, R^{i-2} \pi_{*} \mathbb{Q}\right)=\operatorname{cl}_{X}\left(X_{s}\right) \cup H^{0}\left(C, R^{i-2} \pi_{*} \mathbb{Q}\right)=H^{2}(C, \mathbb{Q}) \cup H^{0}\left(C, R^{i-2} \pi_{*} \mathbb{Q}\right)
$$

и изоморфизм

$$
H^{0}\left(C, R^{i-2} \pi_{*} \mathbb{Q}\right) \stackrel{\cup \mathrm{cl}_{X}\left(X_{s}\right)}{-} \stackrel{-}{\rightarrow} H^{2}\left(C, R^{i-2} \pi_{*} \mathbb{Q}\right)
$$

индуцируется алгебраическим классом $\Delta_{*} \operatorname{cl}_{X}\left(X_{s}\right)$, где $\Delta: X \hookrightarrow X \times X-$ диагональное вложение [14, предложение 1.3.5].

Композищия алгебраических изоморфизмов

$$
\begin{aligned}
\varphi_{i}: H^{0}\left(C, R^{2 d+2-i} \pi_{*} \mathbb{Q}\right) & \stackrel{\left[j_{*} v^{i-2}\right]^{*}}{\longrightarrow} H^{0}\left(C, R^{i-2} \pi_{*} \mathbb{Q}\right) \stackrel{\left[\Delta_{*} \mathrm{cl}_{X}\left(X_{s}\right)\right]^{*}}{\longrightarrow} \\
& ---\longrightarrow H^{2}\left(C, R^{i-2} \pi_{*} \mathbb{Q}\right)
\end{aligned}
$$

является алгебраическим изоморфизмом, определенным алгебраическим классом

$$
\left[\Delta_{*} \mathrm{cl}_{X}\left(X_{s}\right)\right] \circ\left[j_{*} v^{i-2}\right]=\operatorname{pr}_{13 *}\left(\left[j_{*} v^{i-2}\right] \otimes 1_{X} \cup 1_{X} \otimes\left[\Delta_{*} \mathrm{cl}_{X}\left(X_{s}\right)\right]\right) \in H^{2 i}(X \times X, \mathbb{Q}) .
$$

Действительно, очевидно, что

$$
\begin{gathered}
j_{*} v^{i-2} \in H^{2 i-2}(X \times X, \mathbb{Q}), \\
\Delta_{*} \operatorname{cl}_{X}\left(X_{s}\right) \in H^{2+2 \operatorname{dim} X}(X \times X, \mathbb{Q}), \\
{\left[j_{*} v^{i-2}\right] \otimes 1_{X} \cup 1_{X} \otimes\left[\Delta_{*} \operatorname{cl}_{X}\left(X_{s}\right)\right] \in H^{2 i+2 \operatorname{dim} X}(X \times X \times X, \mathbb{Q}),} \\
\operatorname{pr}_{13 *}\left(\left[j_{*} v^{i-2}\right] \otimes 1_{X} \cup 1_{X} \otimes\left[\Delta_{*} \operatorname{cl}_{X}\left(X_{s}\right)\right]\right) \in H^{2 i}(X \times X, \mathbb{Q}) .
\end{gathered}
$$

Преобразуем диаграмму (6.3) в новую диаграмму

$$
\begin{array}{ccccc}
H^{0}\left(C, R^{2 d+2-i} \pi_{*} \mathbb{Q}\right) & \oplus & H^{1}\left(C, R^{2 d+1-i} \pi_{*} \mathbb{Q}\right) & \oplus & H^{2}\left(C, R^{2 d-i} \pi_{*} \mathbb{Q}\right) \\
\simeq \varphi_{i} & & \simeq \downarrow\left[j_{*} v^{i-1}\right]^{*} & & \simeq \downarrow^{\left[j_{*} v^{i}\right]^{*}} \\
H^{2}\left(C, R^{i-2} \pi_{*} \mathbb{Q}\right) & \oplus & H^{1}\left(C, R^{i-1} \pi_{*} \mathbb{Q}\right) & \oplus & H^{2}\left(C, R^{i} \pi_{*} \mathbb{Q}\right),
\end{array}
$$

где все морфизмы - алгебраические изоморфизмы.

Остается осушествить коррекцию морфизма $\left[j_{*} v^{i}\right]^{*}$ в (7.4).

\section{$\S 8$. О компонентах $j_{*} v^{i}$}

\section{1. Лемма. Имеем}

$$
\begin{gathered}
j_{*} v^{i} \in\left[H^{0}\left(C, R^{i} \pi_{*} \mathbb{Q}\right) \otimes H^{2}\left(C, R^{i} \pi_{*} \mathbb{Q}\right)\right] \oplus\left[H^{2}\left(C, R^{i} \pi_{*} \mathbb{Q}\right) \otimes H^{0}\left(C, R^{i} \pi_{*} \mathbb{Q}\right)\right] \\
\oplus\left[H^{1}\left(C, R^{i} \pi_{*} \mathbb{Q}\right) \otimes H^{1}\left(C, R^{i} \pi_{*} \mathbb{Q}\right)\right]
\end{gathered}
$$


ДоКАЗАТЕЛЬСТво. Пусть $\Theta_{n}-$ эндоморфизм $X$, индуцированньй умножением на $n$ в слоях $\pi: X \rightarrow C$, для любого целого числа $n>1$. Хорошо известно, что $H^{a}\left(C, R^{b} \pi_{*} \mathbb{Q}\right)$ совпадает с собственным подпространством $H^{\langle a, b\rangle}(X, \mathbb{Q})$ оператора $\Theta_{n}^{*}$ в $H^{r}(X, \mathbb{Q})$, отвечающим собственному числу $n^{b}[14$, лемма $2 \mathrm{~A} 3]$.

Заметим сначала, что отображение $\Theta_{n}: X \rightarrow X$ является морфизмом схем над $C$, и поэтому оно определяет $C$-морфизм $\Theta_{n} \times_{C} \operatorname{id}_{X}: X \times_{C} X \rightarrow X \times_{C} X$ и $(C \times C)$-морфизм $\Theta_{n} \times \operatorname{id}_{X}: X \times X \rightarrow X \times X$. Более того,

$$
j \circ\left[\Theta_{n} \times_{C} \operatorname{id}_{X}\right]=\left[\Theta_{n} \times \operatorname{id}_{X}\right] \circ j .
$$

Поэтому

$$
j_{*}\left[\Theta_{n} \times_{C} \mathrm{id}_{X}\right]_{*} v^{i}=\left[\Theta_{n} \times \mathrm{id}_{X}\right]_{*} j_{*} v^{i} .
$$

Поскольку $v^{i} \in H^{0}\left(C, R^{i} \pi_{*} \mathbb{Q} \otimes R^{i} \pi_{*} \mathbb{Q}\right)$, то мы имеем

$$
\left[\Theta_{n} \times_{C} \operatorname{id}_{X}\right]^{*} v^{i}=n^{i} v^{i} .
$$

$\mathrm{C}$ другой стороны, $\operatorname{deg}\left[\Theta_{n} \times_{C} \mathrm{id}_{X}\right]=n^{2 d}$. Поэтому из формулы проекции

$$
\left[\Theta_{n} \times_{C} \operatorname{id}_{X}\right]_{*}\left[\Theta_{n} \times_{C} \operatorname{id}_{X}\right]^{*}=\operatorname{deg}\left[\Theta_{n} \times_{C} \operatorname{id}_{X}\right]
$$

получаем соотношения

$$
\begin{aligned}
n^{2 d} v^{i} & =\left[\Theta_{n} \times_{C} \operatorname{id}_{X}\right]_{*}\left[\Theta_{n} \times_{C} \operatorname{id}_{X}\right]^{*} v^{i}=\left[\Theta_{n} \times_{C} \operatorname{id}_{X}\right]_{*}\left(n^{i} v^{i}\right) \\
& =n^{i}\left[\Theta_{n} \times_{C} \operatorname{id}_{X}\right]_{*} v^{i}
\end{aligned}
$$

Видно, что

$$
\left[\Theta_{n} \times_{C} \operatorname{id}_{X}\right]_{*} v^{i}=n^{2 d-i} v^{i}
$$

Следовательно,

$$
n^{2 d-i} j_{*} v^{i}=j_{*}\left(n^{2 d-i} v^{i}\right)=j_{*}\left[\Theta_{n} \times_{C} \mathrm{id}_{X}\right]_{*} v^{i}=\left[\Theta_{n} \times \mathrm{id}_{X}\right]_{*} j_{*} v^{i} .
$$

Пусть $u=j_{*} v^{i}$. Обозначим через $u_{p q r t} \in H^{p}\left(C, R^{q} \pi_{*} \mathbb{Q}\right) \otimes H^{r}\left(C, R^{t} \pi_{*} \mathbb{Q}\right)$ соответствуюшую компоненту $u$. В силу соотношения (3.7)

$$
\left[\Theta_{n} \times \operatorname{id}_{X}\right]^{*} u_{p q r t}=n^{q} u_{p q r t} .
$$

С другой стороны, $\operatorname{deg}\left[\Theta_{n} \times \operatorname{id}_{X}\right]=n^{2 d}$, и из формулы проекции

$$
\left[\Theta_{n} \times \operatorname{id}_{X}\right]_{*}\left[\Theta_{n} \times \operatorname{id}_{X}\right]^{*}=\operatorname{deg}\left[\Theta_{n} \times \operatorname{id}_{X}\right]
$$

следуют соотношения

$$
\begin{aligned}
n^{2 d} u_{p q r t} & =\left[\Theta_{n} \times \operatorname{id}_{X}\right]_{*}\left[\Theta_{n} \times \operatorname{id}_{X}\right]^{*} u_{p q r t}=\left[\Theta_{n} \times \operatorname{id}_{X}\right]_{*}\left(n^{q} u_{p q r t}\right) \\
& =n^{q}\left[\Theta_{n} \times \operatorname{id}_{X}\right]_{*} u_{p q r t} .
\end{aligned}
$$


Поэтому

$$
\left[\Theta_{n} \times \mathrm{id}_{X}\right]_{*} u_{p q r t}=n^{2 d-q} u_{p q r t}
$$

Поскольку

$$
\begin{aligned}
\sum n^{2 d-q} u_{p q r t} & =\left[\Theta_{n} \times \mathrm{id}_{X}\right]_{*} \sum u_{p q r t}=\left[\Theta_{n} \times \mathrm{id}_{X}\right]_{*} j_{*} v^{i} \\
& =n^{2 d-i} j_{*} v^{i}=n^{2 d-i} \sum u_{p q r t}
\end{aligned}
$$

то имеем равенство $q=i$.

Аналогично, используя $\mathrm{id}_{X} \times \Theta_{n}$ вместо $\Theta_{n} \times \mathrm{id}_{X}$, приходим к соотношению $t=i$.

Видно, что $j_{*} v^{i} \in H^{2 i+2}(X \times X, \mathbb{Q})$ принадлежит

$$
\begin{array}{r}
{\left[H^{0}\left(C, R^{i} \pi_{*} \mathbb{Q}\right) \otimes H^{2}\left(C, R^{i} \pi_{*} \mathbb{Q}\right)\right] \oplus\left[H^{2}\left(C, R^{i} \pi_{*} \mathbb{Q}\right) \otimes H^{0}\left(C, R^{i} \pi_{*} \mathbb{Q}\right)\right]} \\
\oplus\left[H^{1}\left(C, R^{i} \pi_{*} \mathbb{Q}\right) \otimes H^{1}\left(C, R^{i} \pi_{*} \mathbb{Q}\right)\right]
\end{array}
$$

Лемма доказана.

8.2. СлЕДСТВИЕ. Для $i=0$ имеем

$$
\begin{aligned}
j_{*} 1=\operatorname{cl}_{X \times X}\left(X \times_{C} X\right) \in\left[H^{0}(C, \mathbb{Q}) \otimes H^{2}(C, \mathbb{Q})\right] \\
\oplus\left[H^{2}(C, \mathbb{Q}) \otimes H^{0}(C, \mathbb{Q})\right] \oplus\left[H^{1}(C, \mathbb{Q}) \otimes H^{1}(C, \mathbb{Q})\right] .
\end{aligned}
$$

8.3. В силу леммы из п. $8.1 j_{*} v^{i-1} \in H^{2 i}(X \times X, \mathbb{Q})$ принадлежит

$$
\begin{array}{r}
{\left[H^{0}\left(C, R^{i-1} \pi_{*} \mathbb{Q}\right) \otimes H^{2}\left(C, R^{i-1} \pi_{*} \mathbb{Q}\right)\right] \oplus\left[H^{2}\left(C, R^{i-1} \pi_{*} \mathbb{Q}\right) \otimes H^{0}\left(C, R^{i-1} \pi_{*} \mathbb{Q}\right)\right]} \\
\oplus\left[H^{1}\left(C, R^{i-1} \pi_{*} \mathbb{Q}\right) \otimes H^{1}\left(C, R^{i-1} \pi_{*} \mathbb{Q}\right)\right] .
\end{array}
$$

Этот алгебраический класс определяет алгебраический изоморфизм

$$
H^{1}\left(C, R^{2 d+1-i} \pi_{*} \mathbb{Q}\right) \stackrel{\left[j_{*} v^{i-1}\right]^{*}}{-}-\underset{\longrightarrow}{\rightarrow} H^{1}\left(C, R^{i-1} \pi_{*} \mathbb{Q}\right)
$$

являющийся композицией

$$
\begin{aligned}
H^{1}\left(C, R^{2 d+1-i} \pi_{*} \mathbb{Q}\right) \stackrel{\stackrel{\mathrm{pr}_{1}^{*}}{\rightarrow}}{\rightarrow} H^{1}\left(C, R^{2 d+1-i} \pi_{*} \mathbb{Q}\right) \otimes H^{0}(C, \mathbb{Q})--\stackrel{j_{*} v^{i-1}}{\rightarrow} \\
---\rightarrow\left[H^{1}\left(C, R^{2 d} \pi_{*} \mathbb{Q}\right) \otimes H^{2}\left(C, R^{i-1} \pi_{*} \mathbb{Q}\right)\right] \\
\\
\quad \oplus\left[H^{2}\left(C, R^{2 d} \pi_{*} \mathbb{Q}\right) \otimes H^{1}\left(C, R^{i-1} \pi_{*} \mathbb{Q}\right)\right] \\
\stackrel{\operatorname{pr}_{2 *}}{\rightarrow} H^{1}\left(C, R^{i-1} \pi_{*} \mathbb{Q}\right) .
\end{aligned}
$$

Наконец, $j_{*} v^{i-2} \in H^{2 i-2}(X \times X, \mathbb{Q})$ принадлежит

$$
\begin{array}{r}
{\left[H^{0}\left(C, R^{i-2} \pi_{*} \mathbb{Q}\right) \otimes H^{2}\left(C, R^{i-2} \pi_{*} \mathbb{Q}\right)\right] \oplus\left[H^{2}\left(C, R^{i-2} \pi_{*} \mathbb{Q}\right) \otimes H^{0}\left(C, R^{i-2} \pi_{*} \mathbb{Q}\right)\right]} \\
\oplus\left[H^{1}\left(C, R^{i-2} \pi_{*} \mathbb{Q}\right) \otimes H^{1}\left(C, R^{i-2} \pi_{*} \mathbb{Q}\right)\right]
\end{array}
$$


Этот алгебраический класс определяет алгебраический изоморфизм

$$
H^{0}\left(C, R^{2 d+2-i} \pi_{*} \mathbb{Q}\right) \stackrel{\left[j_{*} v^{i-2}\right]^{*}}{-} \stackrel{-}{\rightarrow} H^{0}\left(C, R^{i-2} \pi_{*} \mathbb{Q}\right)
$$

являющийся композицией

$$
\begin{aligned}
& H^{0}\left(C, R^{2 d+2-i} \pi_{*} \mathbb{Q}\right) \stackrel{\mathrm{pr}_{1}^{*}}{\rightarrow} H^{0}\left(C, R^{2 d+2-i} \pi_{*} \mathbb{Q}\right) \otimes H^{0}(C, \mathbb{Q}) \stackrel{\cup j_{*} v^{i-2}}{-} \rightarrow \\
& ---\rightarrow\left[H^{0}\left(C, R^{2 d} \pi_{*} \mathbb{Q}\right) \otimes H^{2}\left(C, R^{i-2} \pi_{*} \mathbb{Q}\right)\right] \\
& \oplus\left[H^{2}\left(C, R^{2 d} \pi_{*} \mathbb{Q}\right) \otimes H^{0}\left(C, R^{i-2} \pi_{*} \mathbb{Q}\right)\right] \\
& \oplus\left[H^{1}\left(C, R^{2 d} \pi_{*} \mathbb{Q}\right) \otimes H^{1}\left(C, R^{i-2} \pi_{*} \mathbb{Q}\right)\right] \stackrel{\mathrm{pr}_{2 *}}{\rightarrow} \\
& \stackrel{\mathrm{pr}_{2 *}}{\rightarrow} H^{0}\left(C, R^{i-2} \pi_{*} \mathbb{Q}\right) \text {. }
\end{aligned}
$$

\section{§9. Ограничение линейных расслоений}

9.1. ЛЕмма. Если $\pi: X \rightarrow C$ не имеет нетривиальных постоянных абелевых подсхем, то морфизм ограничения

$$
j^{*}: \operatorname{Pic}^{0}(X \times X) \rightarrow \operatorname{Pic}^{0}\left(X \times_{C} X\right)
$$

многообразий Пикара сюрбективен.

ДокАЗАТЕльСТво. Напомним, что $H^{1}\left(X \times X, \mathscr{O}_{X \times X}\right)$ является касательным пространством в нуле многообразия Пикара $\operatorname{Pic}^{0}(X \times X)$ и $H^{1}\left(X \times_{C} X, \mathscr{O}_{X} \times_{C} X\right)$ касательное пространство в нуле многообразия Пикара $\operatorname{Pic}^{0}\left(X \times_{C} X\right)$. По теории Ходжа

$$
\begin{aligned}
H^{1}(X \times X, \mathbb{Q}) & \otimes \mathbb{C}=H^{1}\left(X \times X, \mathscr{O}_{X \times X}\right) \oplus \overline{H^{1}\left(X \times X, \mathscr{O}_{X \times X}\right)} \\
H^{1}\left(X \times_{C} X, \mathbb{Q}\right) \otimes \mathbb{C} & \left.=H^{1}\left(X \times_{C} X, \mathscr{O}_{X \times_{C} X}\right) \oplus \overline{H^{1}\left(X \times_{C} X, \mathscr{O}_{X} \times_{C} X\right.}\right)
\end{aligned}
$$

Следовательно, остается проверить сюръективность морфизма ограничения

$$
j^{*}: H^{1}(X \times X, \mathbb{Q}) \rightarrow H^{1}\left(X \times_{C} X, \mathbb{Q}\right) .
$$

Спектральная последовательность Лере

$$
E_{2}^{p, q}=H^{p}\left(C \times C, R^{q}(\pi \times \pi)_{*} \mathbb{Q}\right) \Rightarrow H^{*}(X \times X, \mathbb{Q})
$$

индуцирует каноническую точную последовательность $0 \rightarrow E_{2}^{1,0} \rightarrow E^{1} \rightarrow E_{2}^{0,1}$ $[21$, добавление В]. Она имеет вид

$$
0 \rightarrow H^{1}(C \times C, \mathbb{Q}) \rightarrow H^{1}(X \times X, \mathbb{Q}) \rightarrow H^{0}\left(C \times C, R^{1}(\pi \times \pi)_{*} \mathbb{Q}\right) .
$$

Аналогично приходим к точной последовательности

$$
0 \rightarrow H^{1}(C, \mathbb{Q}) \rightarrow H^{1}\left(X \times_{C} X, \mathbb{Q}\right) \rightarrow H^{0}\left(C, R^{1} \tau_{*} \mathbb{Q}\right)
$$


где $\tau: X \times_{C} X \rightarrow C$ - структурный морфизм.

В силу леммы из п. 2.6 имеем следуюшие соотношения:

$$
\begin{aligned}
& H^{0}\left(C \times C, R^{1}(\pi \times \pi)_{*} \mathbb{Q}\right)=H^{0}\left(C, R^{1} \pi_{*} \mathbb{Q}\right) \oplus H^{0}\left(C, R^{1} \pi_{*} \mathbb{Q}\right)=(0), \\
& H^{0}\left(C, R^{1} \tau_{*} \mathbb{Q}\right)=H^{0}\left(C, R^{1} \pi_{*} \mathbb{Q} \otimes \pi_{*} \mathbb{Q}\right) \oplus H^{0}\left(C, \pi_{*} \mathbb{Q} \otimes R^{1} \pi_{*} \mathbb{Q}\right) \\
&=H^{0}\left(C, R^{1} \pi_{*} \mathbb{Q}\right) \oplus H^{0}\left(C, R^{1} \pi_{*} \mathbb{Q}\right)=(0)
\end{aligned}
$$

Пусть $\Delta_{C}: C \rightarrow C \times C$ - диагональное вложение. Коммутативная диаграмма с точными строками

$$
\begin{aligned}
& 0 \rightarrow H^{1}(C \times C, \mathbb{Q}) \rightarrow H^{1}(X \times X, \mathbb{Q}) \rightarrow 0 \\
& \downarrow\left[\Delta_{C}\right]^{*} \rightarrow j^{*} \\
& 0 \rightarrow H^{1}(C, \mathbb{Q}) \quad \rightarrow H^{1}\left(X \times_{C} X, \mathbb{Q}\right) \rightarrow 0
\end{aligned}
$$

показывает, что остается доказать сюръективность канонического морфизма

$$
\left[\Delta_{C}\right]^{*}: H^{1}(C \times C, \mathbb{Q}) \rightarrow H^{1}(C, \mathbb{Q}) .
$$

Поскольку

$$
H^{1}(C \times C, \mathbb{Q})=\left[H^{1}(C, \mathbb{Q}) \otimes H^{0}(C, \mathbb{Q})\right] \oplus\left[H^{0}(C, \mathbb{Q}) \otimes H^{1}(C, \mathbb{Q})\right],
$$

и (по определению $\cup$-произведения) для любых $a, b \in H^{*}(C, \mathbb{Q})$

$$
\left[\Delta_{C}\right]^{*}(a \otimes b)=a \cup b,
$$

то имеем сюръективный морфизм $\left[\Delta_{C}\right]^{*}: H^{1}(C, \mathbb{Q}) \otimes H^{0}(C, \mathbb{Q}) \rightarrow H^{1}(C, \mathbb{Q})$. Лемма доказана.

9.2. Лемма. Если $\pi: X \rightarrow C$ не имеет нетривиальных постоянных абелевых подсхем, то морфизм ограничения

$$
j^{*}: H^{1}\left(X \times X, \mathscr{O}_{X \times X}^{\times}\right) \rightarrow H^{1}\left(X \times_{C} X, \mathscr{O}_{X{ }_{C_{C}}}^{\times}\right)
$$

групп Пикара не является сюрвективным. Более того,

$$
\mathscr{P} \notin j^{*}\left(H^{1}\left(X \times X, \mathscr{O}_{X \times X}^{\times}\right)\right) .
$$

ДоКАЗАТЕЛЬСТво. Предположим, что существует такое линейное расслоение $\mathscr{L}$ на $X \times X$, что $j^{*}(\mathscr{L})=\mathscr{P}$. Пусть $i_{s}: X_{s} \hookrightarrow X-$ каноническое вложение. Тогда имеем канонические вложения

$$
X_{s} \times X_{s} \stackrel{i_{s} \times i_{s}}{\hookrightarrow} X \times_{C} X \stackrel{j}{\hookrightarrow} X \times X .
$$

По теореме Делиня [6, п. 4.1.2] каноническое отображение ограничения

$$
H^{2}(X \times X, \mathbb{Q}) \rightarrow H^{2}\left(X_{s} \times X_{s}, \mathbb{Q}\right)
$$


является композицией канонического сюръективного краевого морфизма

$$
H^{2}(X \times X, \mathbb{Q}) \rightarrow H^{0}\left(C \times C, R^{2}(\pi \times \pi)_{*} \mathbb{Q}\right)
$$

спектральной последовательности Лере $E_{2}^{p, q}=H^{p}\left(C \times C, R^{q}(\pi \times \pi)_{*} \mathbb{Q}\right) \Rightarrow$ $H^{*}(X \times X, \mathbb{Q})$ и канонических отображений

$$
H^{0}\left(C \times C, R^{2}(\pi \times \pi)_{*} \mathbb{Q}\right) \widetilde{\rightrightarrows} H^{2}\left(X_{s} \times X_{s}, \mathbb{Q}\right)^{\pi_{1}(C \times C, s \times s)} \hookrightarrow H^{2}\left(X_{s} \times X_{s}, \mathbb{Q}\right) .
$$

Очевидно, что $\operatorname{cl}_{X \times X}(\mathscr{L}) \in H^{2}(X \times X, \mathbb{Q})$ отображается в элемент

$$
v_{s}=\operatorname{cl}_{X_{s} \times X_{s}}\left(\mathscr{P}_{s}\right) \in H^{2}\left(X_{s} \times X_{s}, \mathbb{Q}\right)^{\pi_{1}(C \times C, s \times s)}
$$

типа Кюннета $(1,1)$, так как $j^{*}\left(\operatorname{cl}_{X \times X}(\mathscr{L})\right)=\operatorname{cl}_{X \times_{C} X}\left(j^{*}(\mathscr{L})\right)=\operatorname{cl}_{X \times_{C} X}(\mathscr{P})$ $[14,1.2 . \mathrm{C}], \quad\left[i_{s} \times i_{s}\right]^{*} \operatorname{cl}_{X \times_{C} X}(\mathscr{P})=\operatorname{cl}_{X_{s} \times X_{s}}\left(\left[i_{s} \times i_{s}\right]^{*}(\mathscr{P})\right)[14,1.2 . \mathrm{C}]$ и $\left[i_{s} \times\right.$ $\left.i_{s}\right]^{*}(\mathscr{P})=\mathscr{P}_{s}$. Поскольку $v_{s}=\operatorname{cl}_{X_{s} \times X_{s}}\left(\mathscr{P}_{s}\right)$ имеет тип Кюннета $(1,1)$, видно, что образ $\mathrm{cl}_{X \times X}(\mathscr{L})$ относительно канонического краевого морфизма

$$
H^{2}(X \times X, \mathbb{Q}) \rightarrow H^{0}\left(C \times C, R^{2}(\pi \times \pi)_{*} \mathbb{Q}\right)
$$

принадлежит

$$
\begin{aligned}
H^{0}\left(C, R^{1} \pi_{*} \mathbb{Q}\right) & \otimes H^{0}\left(C, R^{1} \pi_{*} \mathbb{Q}\right) \underset{\rightarrow}{\hookrightarrow}\left[H^{1}\left(X_{s}, \mathbb{Q}\right) \otimes H^{1}\left(X_{s}, \mathbb{Q}\right)\right]^{\pi_{1}(C \times C, s \times s)} \\
& =\left[H^{1}\left(X_{s}, \mathbb{Q}\right)\right]^{\pi_{1}(C, s)} \otimes\left[H^{1}\left(X_{s}, \mathbb{Q}\right)\right]^{\pi_{1}(C, s)} .
\end{aligned}
$$

В силу леммы из п. $2.6 H^{0}\left(C, R^{1} \pi_{*} \mathbb{Q}\right)=0$. Поэтому $v_{s}=\operatorname{cl}_{X_{s} \times X_{s}}\left(\mathscr{P}_{s}\right)=0$, что противоречит соотношению (6.1). Лемма доказана.

9.3. Лемма. Если $\pi: X \rightarrow C$ не имеет нетривиальных постоянных абелевых подсхем, то морфизм ограничения

$$
j^{*}: H_{\mathrm{alg}}^{2}(X \times X, \mathbb{Q}) \rightarrow H_{\mathrm{alg}}^{2}\left(X \times_{C} X, \mathbb{Q}\right)
$$

не является сюрдективным. Более того,

$$
v \notin j^{*}\left(H_{\mathrm{alg}}^{2}(X \times X, \mathbb{Q})\right) .
$$

ДоКАЗАТЕЛЬСТво. Предположим, что существует такое линейное расслоение $\mathscr{L}$ на $X \times X$, что $j^{*}\left(\operatorname{cl}_{X \times X}(\mathscr{L})\right)=v$.

Очевидно, что ограничение $\operatorname{cl}_{X \times X}(\mathscr{L}) \in H^{2}(X \times X, \mathbb{Q})$ равно элементу

$$
v_{s}=\operatorname{cl}_{X_{s} \times X_{s}}\left(\mathscr{P}_{s}\right) \in H^{2}\left(X_{s} \times X_{s}, \mathbb{Q}\right)^{\pi_{1}(C \times C, s \times s)},
$$

так как $j^{*}\left(\operatorname{cl}_{X \times X}(\mathscr{L})\right)=v$. Поскольку $v_{s}=\operatorname{cl}_{X_{s} \times X_{s}}\left(\mathscr{P}_{s}\right)$ имеет тип Кюннета $(1,1)$, то видно, что образ $\mathrm{cl}_{X \times X}(\mathscr{L})$ относительно канонического краевого морфизма

$$
H^{2}(X \times X, \mathbb{Q}) \rightarrow H^{0}\left(C \times C, R^{2}(\pi \times \pi)_{*} \mathbb{Q}\right)
$$

принадлежит

$$
\begin{aligned}
H^{0}\left(C, R^{1} \pi_{*} \mathbb{Q}\right) & \otimes H^{0}\left(C, R^{1} \pi_{*} \mathbb{Q}\right) \underset{\rightarrow}{\leftrightarrows}\left[H^{1}\left(X_{s}, \mathbb{Q}\right) \otimes H^{1}\left(X_{s}, \mathbb{Q}\right)\right]^{\pi_{1}(C \times C, s \times s)} \\
& =\left[H^{1}\left(X_{s}, \mathbb{Q}\right)\right]^{\pi_{1}(C, s)} \otimes\left[H^{1}\left(X_{s}, \mathbb{Q}\right)\right]^{\pi_{1}(C, s)} .
\end{aligned}
$$

В силу леммы из п. $2.6 H^{0}\left(C, R^{1} \pi_{*} \mathbb{Q}\right)=0$. Поэтому $v_{s}=\operatorname{cl}_{X_{s} \times X_{s}}\left(\mathscr{P}_{s}\right)=0$, что противоречит соотношению (6.1). Лемма доказана. 


\section{§ 10. Циклы Ходжа и условная коррекция морфизма $\left[j_{*} v^{i}\right]^{*}$}

10.1. ТеоремА. Пусть $\pi: X \rightarrow C$ - комплексная абелева схема с главной поляризацией относительной размерности $d$ над гладкой проективной кривой $C$. Тогда для любого натурального числа $i \geqslant 2$ существует единственный цикл Ходжа

$$
\alpha_{i}=\alpha_{i}(X / C) \in\left[H^{0}\left(C, R^{i} \pi_{*} \mathbb{Q}\right) \otimes H^{0}\left(C, R^{i} \pi_{*} \mathbb{Q}\right)\right] \cap H^{i, i}(X \times X, \mathbb{C}),
$$

характеризуемьй следующим условием: $\alpha_{i} \cup \mathrm{cl}_{X \times X}\left(X \times X_{s}\right)$ определяет изоморфизм

$$
H^{2}\left(C, R^{2 d-i} \pi_{*} \mathbb{Q}\right) \stackrel{\leftrightarrows}{\rightarrow} H^{2}\left(C, R^{i} \pi_{*} \mathbb{Q}\right),
$$

совпадающий с алгебрачческим изоморфизмом

$$
\left[j_{*} v^{i}\right]^{*}: H^{2}\left(C, R^{2 d-i} \pi_{*} \mathbb{Q}\right) \stackrel{\rightarrow}{\rightarrow} H^{2}\left(C, R^{i} \pi_{*} \mathbb{Q}\right) .
$$

Если $\alpha_{i}$ алгебраччен, то алгебрачческий класс

$$
\left[\Delta_{*} \mathrm{cl}_{X}\left(X_{s}\right)\right] \circ\left[j_{*} v^{i-2}\right]+j_{*} v^{i-1}+\alpha_{i}
$$

индуцирует алгебраический изоморфизм $H^{2 d+2-i}(X, \mathbb{Q}) \cong H^{i}(X, \mathbb{Q})$ и верна гипотеза $B^{i}(X)$.

ДоКАЗАТЕЛЬСТво. Согласно лемме из п. 8.1

$$
\begin{aligned}
& j_{*} v^{i} \in\left[H^{0}\left(C, R^{i} \pi_{*} \mathbb{Q}\right) \otimes H^{2}\left(C, R^{i} \pi_{*} \mathbb{Q}\right)\right] \oplus\left[H^{2}\left(C, R^{i} \pi_{*} \mathbb{Q}\right) \otimes H^{0}\left(C, R^{i} \pi_{*} \mathbb{Q}\right)\right] \\
& \oplus\left[H^{1}\left(C, R^{i} \pi_{*} \mathbb{Q}\right) \otimes H^{1}\left(C, R^{i} \pi_{*} \mathbb{Q}\right)\right] .
\end{aligned}
$$

В силу соотношения (7.3)

$$
\begin{aligned}
& H^{0}\left(C, R^{i} \pi_{*} \mathbb{Q}\right) \otimes H^{2}\left(C, R^{i} \pi_{*} \mathbb{Q}\right) \\
& \quad=\left[H^{0}\left(C, R^{i} \pi_{*} \mathbb{Q}\right) \otimes H^{0}\left(C, R^{i} \pi_{*} \mathbb{Q}\right)\right] \cup \mathrm{cl}_{X \times X}\left(X \times X_{s}\right), \\
& {\left[H^{2}\left(C, R^{i} \pi_{*} \mathbb{Q}\right) \otimes H^{0}\left(C, R^{i} \pi_{*} \mathbb{Q}\right)\right]} \\
& \quad=\left[H^{0}\left(C, R^{i} \pi_{*} \mathbb{Q}\right) \otimes H^{0}\left(C, R^{i} \pi_{*} \mathbb{Q}\right)\right] \cup \operatorname{cl}_{X \times X}\left(X_{s} \times X\right) .
\end{aligned}
$$

Следовательно, существуют такие однозначно определенные элементы

$$
\begin{aligned}
\alpha_{i}, \beta_{i} & \in H^{0}\left(C, R^{i} \pi_{*} \mathbb{Q}\right) \otimes H^{0}\left(C, R^{i} \pi_{*} \mathbb{Q}\right), \\
\delta_{i} & \in H^{1}\left(C, R^{i} \pi_{*} \mathbb{Q}\right) \otimes H^{1}\left(C, R^{i} \pi_{*} \mathbb{Q}\right),
\end{aligned}
$$

что

$$
j_{*} v^{i}=\alpha_{i} \cup \operatorname{cl}_{X \times X}\left(X \times X_{s}\right)+\beta_{i} \cup \operatorname{cl}_{X \times X}\left(X_{s} \times X\right)+\delta_{i} .
$$

Пусть $V_{\mathbb{Q}}$ - рациональная структура Ходжа. Из общей теории групп мультипликативного типа следует, что определить разложение Ходжа

$$
V_{\mathbb{Q}} \otimes \mathbb{C}=\bigoplus V_{\mathbb{C}}^{p, q}
$$


- это то же самое, что определить морфизм вешественных алгебраических групп $h: \operatorname{Res}_{\mathbb{C} / \mathbb{R}}\left(\mathrm{G}_{m}\right) \rightarrow \mathrm{GL}\left(V_{\mathbb{Q}} \otimes \mathbb{R}\right)$, где $\operatorname{Res}_{\mathbb{C} / \mathbb{R}}\left(\mathrm{G}_{m}\right)$ - вешественная алгебраическая групша, полученная из мультипликативной групшы $\mathrm{G}_{m}$ ограничением поля скаляров от $\mathbb{C}$ до $\mathbb{R}\left[10\right.$, определение В.42]; элемент $z \in \mathbb{C}^{\times}=\operatorname{Res}_{\mathbb{C} / \mathbb{R}}\left(\mathrm{G}_{m}\right)(\mathbb{R})$ действует на $V_{\mathbb{C}}^{p, q}$ как умножение на число $z^{p} \bar{z}^{q}$. Пусть $U^{1}=\left\{z \in \mathbb{C}^{\times}=\operatorname{Res}_{\mathbb{C} / \mathbb{R}}\left(\mathrm{G}_{m}\right)(\mathbb{R}) \mid\right.$ $|z|=1\}$. Наименьшая $\mathbb{Q}$-подгруппа в $\mathrm{GL}\left(V_{\mathbb{Q}}\right)$, группа $\mathbb{R}$-точек которой содержит $h\left(U^{1}\right)$, называется группой Ходжа структуры Ходжа $V_{\mathbb{Q}}$ и обозначается через $\mathrm{Hg}\left(V_{\mathbb{Q}}\right)$. Наименьшая $\mathbb{Q}$-подгруппа в $\mathrm{GL}\left(V_{\mathbb{Q}}\right)$, группа $\mathbb{R}$-точек которой содержит $h\left(\operatorname{Res}_{\mathbb{C} / \mathbb{R}}\left(\mathrm{G}_{m}\right)\right)$, называется группой Мамфорда-Тәйта структуры Ходжа $V_{\mathbb{Q}}$ и обозначается через $\mathrm{MT}\left(V_{\mathbb{Q}}\right)$ [10, определение В.51].

Хорошо известно, что сушествует изогения $\mathrm{G}_{m} \times \mathrm{Hg}\left(V_{\mathbb{Q}}\right) \rightarrow \mathrm{MT}\left(V_{\mathbb{Q}}\right)[10$, лемма В.53]. Если $V_{\mathbb{Q}}$ - поляризуемая рациональная структура Ходжа, то $\mathrm{Hg}\left(V_{\mathbb{Q}}\right)$ и $\mathrm{MT}\left(V_{\mathbb{Q}}\right)$ - редуктивные $\mathbb{Q}$-группы, действуюшие естественным образом на пространстве $V_{\mathbb{Q}}$ [10, предложение В.57].

Важная роль групп Ходжа в анализе структур Ходжа заключается в том, что рациональные $\mathrm{Hg}\left(V_{\mathbb{Q}}\right)$-подпредставления в $V^{\otimes r} \otimes\left(V^{\wedge}\right)^{\otimes s}$ - это в точности рациональные подструктуры Ходжа в $V^{\otimes r} \otimes\left(V^{\wedge}\right)^{\otimes s}[10$, предложение В54].

Предположим, что $V_{\mathbb{Q}}$ - рациональная структура Ходжа четного веса $2 r$. Тогда

$$
V_{\mathbb{Q}}^{\mathrm{Hg}\left(V_{\mathbb{Q}}\right)}=V_{\mathbb{Q}} \cap V_{\mathbb{C}}^{r, r},
$$

где $V_{\mathbb{Q}} \otimes \mathbb{C}=\bigoplus V_{\mathbb{C}}^{p, q}$ - разложение Ходжа [10, следствие В.55]. По определению элементы $V_{\mathbb{Q}}^{\mathrm{Hg}\left(V_{\mathbb{Q}}\right)}=V_{\mathbb{Q}} \cap V_{\mathbb{C}}^{r, r}$ называются ииклами Ходжа.

В рассматриваемой ситуации компоненты

$$
\begin{aligned}
& H^{0}\left(C, R^{i} \pi_{*} \mathbb{Q}\right) \otimes H^{2}\left(C, R^{i} \pi_{*} \mathbb{Q}\right) \\
& \quad=\left[H^{0}\left(C, R^{i} \pi_{*} \mathbb{Q}\right) \otimes H^{0}\left(C, R^{i} \pi_{*} \mathbb{Q}\right)\right] \cup \operatorname{cl}_{X \times X}\left(X \times X_{s}\right), \\
& \quad\left[H^{2}\left(C, R^{i} \pi_{*} \mathbb{Q}\right) \otimes H^{0}\left(C, R^{i} \pi_{*} \mathbb{Q}\right)\right] \\
& \quad=\left[H^{0}\left(C, R^{i} \pi_{*} \mathbb{Q}\right) \otimes H^{0}\left(C, R^{i} \pi_{*} \mathbb{Q}\right)\right] \cup \mathrm{cl}_{X \times X}\left(X_{s} \times X\right), \\
& H^{1}\left(C, R^{i} \pi_{*} \mathbb{Q}\right) \otimes H^{1}\left(C, R^{i} \pi_{*} \mathbb{Q}\right)
\end{aligned}
$$

являются рациональными подструктурами Ходжа в $H^{2 i+2}(X \times X, \mathbb{Q})$ (напомним, что $H^{0}\left(C, R^{i} \pi_{*} \mathbb{Q}\right)$ - рациональная подструктура Ходжа в $H^{i}(X, \mathbb{Q})$, канонически отождествляемая (в силу (3.2)) с собственным подпространством оператора $\left[\Theta_{n}\right]^{*}$ на $H^{i}(X, \mathbb{Q})$, отвечающим собственному числу $n^{i}$; аналогично $H^{1}\left(C, R^{i} \pi_{*} \mathbb{Q}\right)$ канонически отождествляется с собственным подпространством оператора $\left[\Theta_{n}\right]^{*}$ на $H^{i+1}(X, \mathbb{Q})$, отвечающим собственному числу $\left.n^{i}\right)$. Следовательно, они стабильны относительно действия $\mathrm{Hg}\left(H^{2 i+2}(X \times X, \mathbb{Q})\right)$.

По теореме Лефшеца

$$
\mathrm{NS}(X \times X) \otimes \mathbb{Q} \underset{\rightrightarrows}{\rightarrow} H^{2}(X \times X, \mathbb{Q}) \cap H^{1,1}(X \times X, \mathbb{C}) .
$$

В силу соотношения (10.1)

$$
\mathrm{NS}(X \times X) \otimes \mathbb{Q} \stackrel{\longrightarrow}{\rightarrow} H^{2}(X \times X, \mathbb{Q})^{\operatorname{Hg}\left(H^{2}(X \times X, \mathbb{Q})\right)}
$$


Поэтому $\operatorname{cl}_{X \times X}\left(X \times X_{s}\right)$ и $\mathrm{cl}_{X \times X}\left(X_{s} \times X\right)$ неподвижны при каноническом действии $U^{1}$ на $H^{2}(X \times X, \mathbb{C})$. Аналогично, алгебраический класс $j_{*} v^{i}$ принадлежит

$$
\begin{aligned}
H_{\text {alg }}^{2 i+2}(X \times X, \mathbb{Q}) & \hookrightarrow H^{2 i+2}(X \times X, \mathbb{Q}) \cap H^{i+1, i+1}(X \times X, \mathbb{C}) \\
& =H^{2 i+2}(X \times X, \mathbb{Q})^{\operatorname{Hg}\left(H^{2 i+2}(X \times X, \mathbb{Q})\right)}
\end{aligned}
$$

и неподвижен при действии $U^{1}$ на $H^{2 i+2}(X \times X, \mathbb{C})$. Поскольку это действие совместимо с $\cup$-произведением, то элементы

$$
\alpha_{i}, \beta_{i} \in H^{0}\left(C, R^{i} \pi_{*} \mathbb{Q}\right) \otimes H^{0}\left(C, R^{i} \pi_{*} \mathbb{Q}\right)
$$

неподвижны при действии $U^{1}$ на $H^{2 i}(X \times X, \mathbb{C})$. Следовательно, $\alpha_{i}$ и $\beta_{i}$ неподвижны при действии группы Ходжа $\operatorname{Hg}\left(H^{2 i}(X \times X, \mathbb{Q})\right)$. Другими словами, $\alpha_{i}$ и $\beta_{i}-$ циклы Ходжа,

$$
\alpha_{i}, \beta_{i} \in\left[H^{0}\left(C, R^{i} \pi_{*} \mathbb{Q}\right) \otimes H^{0}\left(C, R^{i} \pi_{*} \mathbb{Q}\right)\right] \cap H^{i, i}(X \times X, \mathbb{C}) .
$$

Утверждаем, что цикл Ходжа $\alpha_{i} \cup \mathrm{cl}_{X \times X}\left(X \times X_{s}\right)$ индуцирует алгебрачческий изоморфизм

$$
\left[j_{*} v^{i}\right]^{*}: H^{2}\left(C, R^{2 d-i} \pi_{*} \mathbb{Q}\right) \stackrel{\Im}{\rightarrow} H^{2}\left(C, R^{i} \pi_{*} \mathbb{Q}\right)
$$

и цикл Ходжа $\alpha_{i}$ индуцирует изоморфизм

$$
H^{2}\left(C, R^{2 d-i} \pi_{*} \mathbb{Q}\right) \stackrel{\rightrightarrows}{\rightarrow} H^{0}\left(C, R^{i} \pi_{*} \mathbb{Q}\right),
$$

который не предполагается алгебраическим.

Действительно, в силу (6.2) алгебраический класс $j_{*} v^{i}$ индуцирует алгебраический изоморфизм

$$
H^{2}\left(C, R^{2 d-i} \pi_{*} \mathbb{Q}\right) \stackrel{\Im}{\rightarrow} H^{2}\left(C, R^{i} \pi_{*} \mathbb{Q}\right),
$$

определенный композицией

$$
\begin{aligned}
H^{2}\left(C, R^{2 d-i} \pi_{*} \mathbb{Q}\right) \stackrel{\mathrm{pr}_{1}^{*}}{\rightarrow} & H^{2}\left(C, R^{2 d-i} \pi_{*} \mathbb{Q}\right) \otimes H^{0}(C, \mathbb{Q}) \\
& --\frac{\cup j_{*} v^{i}}{\rightarrow} H^{2 d+4+i}(X \times X, \mathbb{Q}) \stackrel{\mathrm{pr}_{2 *}}{\rightarrow} H^{i+2}(X, \mathbb{Q}) .
\end{aligned}
$$

Поскольку элемент $\beta_{i} \cup \mathrm{cl}_{X \times X}\left(X_{s} \times X\right)+\delta_{i}$ аннулирует $H^{2}\left(C, R^{2 d-i} \pi_{*} \mathbb{Q}\right) \otimes$ $H^{0}(C, \mathbb{Q})$, то алгебраический изоморфизм $(10.2)$ совпадает с композищией

$$
\begin{aligned}
& H^{2}\left(C, R^{2 d-i} \pi_{*} \mathbb{Q}\right) \stackrel{\mathrm{pr}_{1}^{*}}{\rightarrow} H^{2}\left(C, R^{2 d-i} \pi_{*} \mathbb{Q}\right) \otimes H^{0}(C, \mathbb{Q})
\end{aligned}
$$

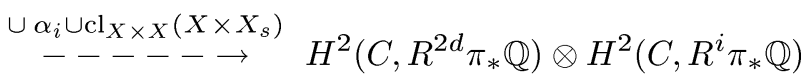

$$
\begin{aligned}
& \stackrel{\mathrm{pr}_{2 *}}{\rightarrow} H^{2}\left(C, R^{i} \pi_{*} \mathbb{Q}\right) \text {. }
\end{aligned}
$$


В частности, отображение

$$
H^{2}\left(C, R^{2 d-i} \pi_{*} \mathbb{Q}\right) \otimes H^{0}(C, \mathbb{Q}) \stackrel{\cup \alpha_{i}}{\rightarrow} H^{2}\left(C, R^{2 d} \pi_{*} \mathbb{Q}\right) \otimes H^{0}\left(C, R^{i} \pi_{*} \mathbb{Q}\right)
$$

инъективно.

С другой стороны, (7.3) и (10.2) влекут соотношения

$$
\operatorname{dim} H^{2}\left(C, R^{2 d-i} \pi_{*} \mathbb{Q}\right)=\operatorname{dim} H^{2}\left(C, R^{i} \pi_{*} \mathbb{Q}\right)=\operatorname{dim} H^{0}\left(C, R^{i} \pi_{*} \mathbb{Q}\right) .
$$

Следовательно, отображение (10.3) биективно, и утверждение доказано.

Если $\alpha_{i}$ алгебраичен, то он индуцирует алгебраический изоморфизм

$$
H^{2}\left(C, R^{2 d-i} \pi_{*} \mathbb{Q}\right) \Im H^{0}\left(C, R^{i} \pi_{*} \mathbb{Q}\right),
$$

поэтому существует алгебраическая коррекция

$$
\begin{array}{ccccc}
H^{0}\left(C, R^{2 d+2-i} \pi_{*} \mathbb{Q}\right) & \oplus & H^{1}\left(C, R^{2 d+1-i} \pi_{*} \mathbb{Q}\right) & \oplus & H^{2}\left(C, R^{2 d-i} \pi_{*} \mathbb{Q}\right) \\
\simeq \downarrow^{\varphi_{i}} & & \simeq \downarrow^{\left[j v^{i-1}\right]^{*}} & & \simeq \downarrow^{\left[\alpha_{i}\right]^{*}} \\
H^{2}\left(C, R^{i-2} \pi_{*} \mathbb{Q}\right) & \oplus & H^{1}\left(C, R^{i-1} \pi_{*} \mathbb{Q}\right) & \oplus & H^{0}\left(C, R^{i} \pi_{*} \mathbb{Q}\right)
\end{array}
$$

морфизма $\left[j_{*} v^{i}\right]^{*}$ в диаграмме $(7.4)$, и гипотеза $B^{i}(X)$ верна. Теорема доказана.

10.2. СледСТвиЕ. Если иикль Ходжка $\alpha_{i}(X / C)$ алгебраические для всех натуральных чисел $i \geqslant 2$ и для всех комплексных абелевых схем с главной поляризацией над гладкими проективньми кривыми $\pi: X \rightarrow C$ без нетривиальных постоянных абелевых подсхем, то гипотеза Ходжа верна для всех абелевых многообразий.

ДокАЗАТЕЛЬСтво. По теореме из п. 10.1 гипотеза $B^{i}(X)$ верна для всех $i \geqslant 2$. Поскольку $B^{0}(X)$ и $B^{1}(X)$ выполняются в силу результатов п. 6.7 , то гипотеза $B^{i}(X)$ верна для всех $i \geqslant 0$. Остается применить теорему Андре из п. 2.1, следствие из п. 2.3 и замечание из п. 2.7.

\section{$\S 11$. Доказательство гипотезы $B^{i}(X)$ для некоторых абелевых схем}

11.1. Пусть $\pi: X \rightarrow C$ - комплексная абелева схема относительной размерности $d$ над гладкой проективной кривой. Существует такое конечно порожденное расширение $k$ поля $\mathbb{Q}$, что $X, C, \pi$ определены над $k \hookrightarrow \mathbb{C}$ :

$$
X=X_{0} \otimes_{k} \mathbb{C}, \quad C=C_{0} \otimes_{k} \mathbb{C}, \quad \pi=\pi_{0} \otimes_{k} \mathbb{C} .
$$

Пусть $X_{0 \eta}$ - обший слой абелевой схемы $\pi_{0}: X_{0} \rightarrow C_{0}$, и пусть $X_{0 \bar{\eta}}=X_{0 \eta} \otimes_{k(\eta)} \mathbb{C}$ для некоторого вложения $k(\eta) \hookrightarrow \mathbb{C}$, продолжающего вложение $k \hookrightarrow \mathbb{C}$.

Назовем $X_{0 \bar{\eta}}$ общим геометрическим слоем абелевой схемы $\pi_{0}: X_{0} \rightarrow C_{0}$.

Для любого комплексного абелева многообразия $X_{s}$ обозначим через $\operatorname{Hg}\left(X_{s}\right) \stackrel{\text { def }}{=} \mathrm{Hg}\left(H^{1}\left(X_{s}, \mathbb{Q}\right)\right)$ әруппу Ходжа $X_{s}$. Это редуктивная связная алгебраическая $\mathbb{Q}$-группа с каноническим действием на $H^{*}\left(X_{s}, \mathbb{Q}\right)=\wedge^{*} H^{1}\left(X_{s}, \mathbb{Q}\right)$. В силу соотношения (10.1)

$$
H^{2 i}\left(X_{s}, \mathbb{Q}\right)^{\operatorname{Hg}\left(X_{s}\right)}=H^{2 i}\left(X_{s}, \mathbb{Q}\right) \cap H^{i, i}\left(X_{s}, \mathbb{C}\right) .
$$


11.2. ТЕОРЕМА. Предположим, что для комплексной абелевой схемы $\pi: X \rightarrow C$ относительной размерности d над гладкой проективной кривой $C$ $\operatorname{Lie} \operatorname{Hg}\left(X_{0 \bar{\eta}}\right)$ является простой алгеброй Ли типа $C_{d}$.

Тогда гипотеза $B(X)$ верна. $B$ частности, операторы ${ }^{{ }} *, *,{ }^{\mathrm{c}} \Lambda, \Lambda$ на $H^{*}(X, \mathbb{Q})$ являются алгебрачческими, численная әквивалентность алгебраических чиклов на $X$ совпадает с гомологической эквивалентностью, компоненты Кюннета произвольного алгебрачческого чикла на $X \times X$ алгебраичесжие.

ДокАЗАТЕЛЬСТво. Пусть $\rho: \pi_{1}(C, s) \rightarrow \mathrm{GL}\left(H^{1}\left(X_{s}, \mathbb{Q}\right)\right)$ - каноническое представление монодромии. Обозначим через $G$ замыкание $\rho\left(\pi_{1}(C, s)\right)$ в топологии Зариского группы $\mathrm{GL}\left(H^{1}\left(X_{s}, \mathbb{Q}\right)\right)$. По определению

$$
H^{i}\left(X_{s}, \mathbb{Q}\right)^{G}=H^{i}\left(X_{s}, \mathbb{Q}\right)^{\pi_{1}(C, s)}
$$

(см. $[34, \S 7.0])$.

В силу теоремы из п. 1.6 можно рассматривать разветвленное накрытие $C^{\prime} \rightarrow C$ и абелеву схему $X^{\prime}=X \times_{C} C^{\prime} \rightarrow C^{\prime}$ вместо $X \rightarrow C$. Можно считать, что $G-$ связная $\mathbb{Q}$-группа. Эта группа полупроста $[6$, следствие 4.2.9].

По теореме Делиня для всех $s$ вне счетного подмножества кривой $C$ группа $G$ является нормальной подгруппой группы Ходжа $\operatorname{Hg}\left(X_{s}\right)$ [7, предложение 7.5$]$; [34, теорема 7.3]. В частности, сушествует вложение $G \hookrightarrow \operatorname{Hg}\left(X_{0 \bar{\eta}}\right)$.

Если $G=1$, то абелева схема $\pi: X \rightarrow C$ постоянна [6, 4.1.3.3], $[7, \S 5.2]:$ сушествует такое абелево многообразие $J$, что $X \widetilde{\rightarrow} J \times C$. Поскольку $B(C)$ верна и $B(J)$ выполняется по теореме Либермана (следствие из п. 1.8), то гипотеза $B(X)$ верна в силу свойства (1.10).

Следовательно, можно считать что $G$ - нетривиальная нормальная алгебраическая подгруппа простой алгебраической группы $\mathrm{Hg}\left(X_{0 \bar{\eta}}\right)$, и поэтому

$$
\operatorname{Lie} G=\operatorname{Lie} \operatorname{Hg}\left(X_{0 \bar{\eta}}\right)
$$

- простая алгебра Ли типа $C_{d}$.

Имеем

$$
\begin{aligned}
H^{0}\left(C, R^{i} \pi_{*} \mathbb{Q}\right) & \stackrel{\rightrightarrows}{\rightarrow} H^{i}\left(X_{s}, \mathbb{Q}\right)^{\pi_{1}(C, s)}=H^{i}\left(X_{s}, \mathbb{Q}\right)^{G} \\
& =H^{i}\left(X_{s}, \mathbb{Q}\right)^{\operatorname{Lie} G}=H^{i}\left(X_{s}, \mathbb{Q}\right)^{\operatorname{sp}\left(H^{1}\left(X_{s}, \mathbb{Q}\right)\right)} \\
& =\left[\wedge^{i} H^{1}\left(X_{s}, \mathbb{Q}\right)\right]^{\operatorname{sp}\left(H^{1}\left(X_{s}, \mathbb{Q}\right)\right)} .
\end{aligned}
$$

С другой стороны, пусть $\Gamma$ - образующая одномерного пространства $\left[\wedge^{2} H^{1}\left(X_{s}, \mathbb{Q}\right)\right]^{\operatorname{sp}\left(H^{1}\left(X_{s}, \mathbb{Q}\right)\right)}$. Тогда

$$
\left[\wedge^{i} H^{1}\left(X_{s}, \mathbb{Q}\right)\right]^{\operatorname{sp}\left(H^{1}\left(X_{s}, \mathbb{Q}\right)\right)}= \begin{cases}0 & \text { для нечетного } i, \\ \mathbb{Q} \cdot \Gamma^{\wedge i / 2} & \text { для четного } i\end{cases}
$$

(см. [28, лемма 4.8.4]). 
Следовательно,

$$
H^{0}\left(C, R^{i} \pi_{*} \mathbb{Q}\right)= \begin{cases}0 & \text { для нечетного } i, \\ \mathbb{Q} \cdot h^{\cup i / 2} & \text { для четного } i,\end{cases}
$$

где $h$ - образ класса $\mathrm{cl}_{X}(H)$ гиперплоского сечения при каноническом сюръективном краевом морфизме $H^{2}(X, \mathbb{Q}) \rightarrow H^{0}\left(C, R^{2} \pi_{*} \mathbb{Q}\right)$ спектральной последовательности Лере $E_{2}^{p, q}=H^{p}\left(C, R^{q} \pi_{*} \mathbb{Q}\right) \Rightarrow H^{*}(X, \mathbb{Q})$.

Мы видим, что $H^{0}\left(C, R^{i} \pi_{*} \mathbb{Q}\right) \otimes H^{0}\left(C, R^{i} \pi_{*} \mathbb{Q}\right)$ тривиально или порождено алгебраическим классом $h^{\cup i / 2} \otimes h^{\cup i / 2}$, поэтому

$$
\alpha_{i}(X / C) \in H^{0}\left(C, R^{i} \pi_{*} \mathbb{Q}\right) \otimes H^{0}\left(C, R^{i} \pi_{*} \mathbb{Q}\right)
$$

является алгебраическим, и теорема следует из теоремы п. 10.1.

11.3. СлеДСтвИЕ. Предположим, ито $\operatorname{End}\left(X_{s}\right)=\mathbb{Z}$ для некоторого геометрического слоя комплексной абелевой схемы $\pi: X \rightarrow C$ нечетной относительной размерности d над гладкой проективной кривой $C$.

Тогда гипотеза $B(X)$ верна.

Действительно,

$$
\operatorname{End}\left(X_{0 \bar{\eta}}\right) \hookrightarrow \operatorname{End}\left(X_{s}\right)=\mathbb{Z} .
$$

Поэтому $\operatorname{End}\left(X_{0 \bar{\eta}}\right)=\mathbb{Z}$. Поскольку $d$ нечетно, то $\operatorname{Hg}\left(X_{0 \bar{\eta}}\right)$ - простая алгебраическая $\mathbb{Q}$-группа типа $C_{d}[28$, теорема 4.7$],[29$, теорема 6.2].

11.4. СЛЕДСТВИЕ. Пусть

$$
\operatorname{Ex}(1)=\{4,10,16,32,64,108,126,256,500,512,864,1024, \ldots\}
$$

- множество исключительных чисел в смысле [31] для простых абелевых многообразий типа I по классификации Альберта. $\operatorname{Ecли~} \operatorname{End}\left(X_{s}\right)=\mathbb{Z}$ для некоторого геометрического слоя комплексной абелевой схемы $\pi: X \rightarrow C$ относительной размерности $d \notin \operatorname{Ex}(1)$ над гладкой проективной кривой $C$, то гипотеза $B(X)$ верна.

Действительно, известно, что в рассматриваемом случае $\mathrm{Hg}\left(X_{0 \bar{\eta}}\right)$ - простая алгебраическая $\mathbb{Q}$-группа типа $C_{d}[31$, теорема 1.1$]$.

11.5. СЛЕДСтВИЕ. Если $\operatorname{End}\left(X_{s}\right)=\mathbb{Z}$ для некоторого геометрического слоя комплексной абелевой схемь $\pi: X \rightarrow C$ относительной размерности $d \in\{4,10\}$ над гладкой проективной кривой $C$, то гипотеза $B(X)$ верна.

Действительно, известно, что в рассматриваемом случае $\mathrm{Hg}\left(X_{0 \bar{\eta}}\right)$ - простая алгебраическая $\mathbb{Q}$-группа типа $C_{d}$ или типа $A_{1} \times A_{1} \times A_{1}(d=4)($ над $\overline{\mathbb{Q}}), A_{5}$ $(d=10)$. Поэтому $G=1$ или $G$-простая алгебраическая $\mathbb{Q}$-группа того же типа, что и групша $\operatorname{Hg}\left(X_{0 \bar{\eta}}\right)$, и

$$
H^{0}\left(C, R^{i} \pi_{*} \mathbb{Q}\right)= \begin{cases}(0) & \text { для нечетного } i, \\ \mathbb{Q} \cdot h^{\cup i / 2} & \text { для четного } i\end{cases}
$$

(см. [31, лемма 2.6]), $H^{0}\left(C, R^{i} \pi_{*} \mathbb{Q}\right) \otimes H^{0}\left(C, R^{i} \pi_{*} \mathbb{Q}\right)$ тривиально или порождено алгебраическим классом $h^{\cup i / 2} \otimes h^{\cup i / 2}$. Остается применить теорему из п. 10.1. 
11.6. ТЕОРемА. Предположим, что для комплексной абелевой схемы $\pi: X \rightarrow C$ относительной размерности $d$ над гладкой проективной кривой $C$ $\operatorname{End}\left(X_{0 \bar{\eta}}\right) \otimes \mathbb{Q}$ - мнимое квадратичное расиирение поля $\mathbb{Q}$ и полупростая часть $\left[\operatorname{Lie~} \mathrm{Hg}\left(X_{0 \bar{\eta}}\right)\right]^{\mathrm{ss}}$ редуктивной алгебры Ли $\operatorname{Lie} \mathrm{Hg}\left(X_{0 \bar{\eta}}\right)$ является простой алгеброй Ли типа $A_{d-1}$.

Тогда для любого $i \neq d$ изоморфизм

$$
\Lambda^{d+1-i}: H^{2 d+2-i}(X, \mathbb{Q}) \stackrel{\rightarrow}{\rightarrow} H^{i}(X, \mathbb{Q})
$$

является алгебраическим.

ДокАЗАТЕЛЬСТво. Поскольку $G$ - нормальная полупростая подгруппа в $\mathrm{Hg}\left(X_{0 \bar{\eta}}\right)$, то она является нормальной подгрупшпой в $\left[\mathrm{Hg}\left(X_{0 \bar{\eta}}\right)\right]^{\text {ss }}$. Поэтому $G=1$ (в этой ситуации абелева схема $\pi: X \rightarrow C$ постоянна и $B(X)$ верна) или $G$-простая $\mathbb{Q}$-группа типа $A_{d-1}$. В последнем случае

$$
H^{1}\left(X_{s}, \mathbb{Q}\right) \otimes \overline{\mathbb{Q}}=E\left(\omega_{1}\right) \oplus E\left(\omega_{1}\right)^{*}=E\left(\omega_{1}\right) \oplus E\left(\omega_{d-1}\right)
$$

- сумма неприводимых представлений $V=E\left(\omega_{1}\right)$ и $V^{*}=E\left(\omega_{d-1}\right)$ со старшими весами $\omega_{1}$ и $\omega_{d-1}$ простой алгебры Ли $g=[\operatorname{Lie} G] \otimes \overline{\mathbb{Q}}$ типа $A_{d-1}[31, \S 2.9]$.

Если $i \leqslant d-1$, то

$$
\begin{aligned}
{\left[H^{i}\left(X_{s}, \mathbb{Q}\right) \otimes \overline{\mathbb{Q}}\right]^{g} } & =\wedge^{i}\left(V \oplus V^{*}\right)^{g}=\wedge^{i}\left(V \oplus V^{*}\right)^{\mathrm{sl}(V)} \\
& =\bigoplus_{m=0}^{i}\left[\wedge^{m} V \otimes \wedge^{i-m} V^{*}\right]^{\mathrm{sl}(V)}=\bigoplus_{m=0}^{i} \operatorname{Hom}_{\mathrm{sl}(V)}\left(\wedge^{i-m} V, \wedge^{m} V\right) .
\end{aligned}
$$

Напомним, что

$$
\wedge^{m} V=\wedge^{m} E\left(\omega_{1}\right)=E\left(\omega_{m}\right)
$$

- неприводимое представление в случае $d-1 \geqslant m \geqslant 1$ и

$$
\wedge^{i-m} V=\wedge^{i-m} E\left(\omega_{1}\right)=E\left(\omega_{i-m}\right)
$$

- неприводимое представление в случае $d-1 \geqslant i-m \geqslant 1$ [3, гл. 8, § 13.1]. Следовательно, по лемме Шура

$$
\operatorname{Hom}_{\mathrm{sl}(V)}\left(\wedge^{i-m} V, \wedge^{m} V\right) \neq(0) \Leftrightarrow i-m=m \quad(\text { для } d-1 \geqslant i \geqslant i-m \geqslant 1)
$$

$\mathrm{c}$

$$
\operatorname{dim}\left[\wedge^{m} V \otimes \wedge^{m} V^{*}\right]^{\mathrm{sl}(V)}=1 .
$$

Если $d-1 \geqslant i \geqslant i-m=0$, то

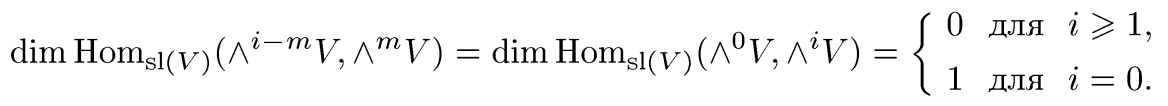

В частности, для всех $i \leqslant d-1$

$$
\operatorname{dim}\left[H^{i}\left(X_{s}, \mathbb{Q}\right)\right]^{G}=\operatorname{dim}\left[H^{i}\left(X_{s}, \mathbb{Q}\right)\right]^{\pi_{1}(C, s)}= \begin{cases}0 & \text { для нечетного } i, \\ 1 & \text { для четного } i .\end{cases}
$$


Случай $i>d$ сводится к предыдушему с помощью сильной теоремы Лефшеца. Видно, что для $i \neq d$

$$
H^{0}\left(C, R^{i} \pi_{*} \mathbb{Q}\right)= \begin{cases}(0) & \text { для нечетного } i, \\ \mathbb{Q} \cdot h^{\cup i / 2} & \text { для четного } i,\end{cases}
$$

$H^{0}\left(C, R^{i} \pi_{*} \mathbb{Q}\right) \otimes H^{0}\left(C, R^{i} \pi_{*} \mathbb{Q}\right)$ тривиально илипорождается алгебраическим клас$\operatorname{coм} h^{\cup i / 2} \otimes h^{\cup i / 2}$. Остается применить теорему из п. 10.1.

Наконец, для $i=d$ имеем

$$
\begin{aligned}
{\left[H^{d}\left(X_{s}, \mathbb{Q}\right)\right.} & \otimes \overline{\mathbb{Q}}]^{g}=\wedge^{d}\left(V \oplus V^{*}\right)^{g}=\wedge^{d}\left(V \oplus V^{*}\right)^{\mathrm{sl}(V)} \\
& =\bigoplus_{m=1}^{d-1}\left[\wedge^{m} V \otimes \wedge^{d-m} V^{*}\right]^{\mathrm{sl}(V)} \oplus\left[\wedge^{0} V \otimes \wedge^{d} V^{*}\right] \oplus\left[\wedge^{d} V \otimes \wedge^{0} V^{*}\right] \\
& =\bigoplus_{m=1}^{d-1} \operatorname{Hom}_{\mathrm{sl}(V)}\left(\wedge^{d-m} V, \wedge^{m} V\right) \oplus\left[\wedge^{0} V \otimes \wedge^{d} V^{*}\right] \oplus\left[\wedge^{d} V \otimes \wedge^{0} V^{*}\right]
\end{aligned}
$$

и

$$
\operatorname{dim}\left[H^{d}\left(X_{s}, \mathbb{Q}\right)\right]^{G}=\operatorname{dim}\left[H^{d}\left(X_{s}, \mathbb{Q}\right)\right]^{\pi_{1}(C, s)}= \begin{cases}2 & \text { для нечетного } d \\ 3 & \text { для четного } d .\end{cases}
$$

Теорема доказана.

11.7. СледСТВИЕ. Пусть

$$
\begin{gathered}
\operatorname{Ex}(4)=\{9,10,15,16,21,25,27,28,35,36,45,49, \\
55,56,64,66,78,81,84,91,100, \ldots\}
\end{gathered}
$$

- множество исключительных чисел в смысле [31] для простых абелевых многообразий типа IV по классификации Альберта. $\operatorname{Ecли~} \operatorname{End}_{C}(X) \otimes \mathbb{Q} u$ $\operatorname{End}\left(X_{s}\right) \otimes \mathbb{Q}$ - мнимье квадратичные расширения $\mathbb{Q}$, где $X_{s}$ - некоторый геометрический слой комплексной абелевой схемы $\pi: X \rightarrow C$ относительной размерности $d \notin \operatorname{Ex}(4)$ над гладкой проективной кривой $C$, то для любого $i \neq d$ изоморфизм

$$
\Lambda^{d+1-i}: H^{2 d+2-i}(X, \mathbb{Q}) \stackrel{\rightrightarrows}{\rightarrow} H^{i}(X, \mathbb{Q})
$$

является алгебраическим.

Действительно, известно, что в рассматриваемом случае $\mathrm{Hg}\left(X_{0 \bar{\eta}}\right)$ - редуктивная алгебраическая $\mathbb{Q}$-группа с полупростой частью $\left[\operatorname{Hg}\left(X_{0 \bar{\eta}}\right)\right]^{\text {ss }}$ типа $A_{d-1}[31$, теорема 1.1].

11.8. Теорема. Пусть $\pi: X \rightarrow C$ - абелева схема над гладкой проективной кривой $C$. Предположим, что некоторый геометрический слой $X_{s}$ является простымм абелевым многообразием простой размерности $d$.

Тогда для любого $i \neq d$ изоморфизм

$$
\Lambda^{d+1-i}: H^{2 d+2-i}(X, \mathbb{Q}) \underset{\rightarrow}{\rightarrow} H^{i}(X, \mathbb{Q})
$$

является алгебраическим. 
ДокАЗАТЕЛЬСТВо. В силу замечания из п. 2.7 можно считать, что абелева схема $\pi: X \rightarrow C$ не содержит нетривиальных постоянных абелевых подсхем. Поскольку $X_{s}$ - простое абелево многообразие, то слой $X_{0 \bar{\eta}}$ является простым абелевым многообразием простой размерности $d$.

Хорошо известно, что $\operatorname{End}\left(X_{0 \bar{\eta}}\right) \otimes \mathbb{Q}$ изоморфно вполне вешественному расширению $K_{0}$ поля $\mathbb{Q}$ степени $\left[K_{0}: \mathbb{Q}\right] \in\{1, d\}$ или мнимому квадратичному расширению $K$ вполне вещественного поля $K_{0}$ степени $[K: \mathbb{Q}] \in\{2,2 d\}[29,6.1]$.

Предположим, что $\operatorname{End}\left(X_{0 \bar{\eta}}\right) \otimes \mathbb{Q}=K_{0}$. Если $K_{0}=\mathbb{Q}$, то мы имеем ситуацию, рассмотренную в следствиях из пा. 11.3 и 11.4 , и гипотеза $B(X)$ верна.

Если $K_{0} \neq \mathbb{Q}$, то $K_{0}$ - вполне вешественное поле степени $\left[K_{0}: \mathbb{Q}\right]=d$. Поскольку представление монодромии

$$
\rho: \pi_{1}(C, s) \rightarrow \mathrm{GL}\left(H^{1}\left(X_{s}, \mathbb{Q}\right)\right)
$$

полупросто $[6,4.2 .6]$, то $\mathbb{Q}$-алгебра $H=\operatorname{End}\left(R^{1} \pi_{*} \mathbb{Q}\right)$ изоморфна прямой сумме алгебр вида $M_{n_{\alpha}}\left(D_{\alpha}\right)$, где $D_{\alpha}$ - алгебра с делением над $\mathbb{Q}$. С другой стороны, центр $Z$ алгебры $H$ имеет тип Ходжа $(0,0)$ и, следовательно, содержится в центре алгебры с делением $\operatorname{End}_{C}(X) \otimes \mathbb{Q} \hookrightarrow \operatorname{End}\left(X_{0 \bar{\eta}}\right) \otimes \mathbb{Q}=K_{0}[6,4.4 .7]$. Поэтому $H \widetilde{\neg} M_{n}(D)$, где $D$ - центральная алгебра с делением над вполне вещественным полем $Z \hookrightarrow K_{0}$. Пусть $e=\operatorname{dim}_{\mathbb{Q}} Z$ и $f^{2}=\operatorname{dim}_{Z} D$. Пространство $H^{1}\left(X_{s}, \mathbb{Q}\right)$ разлагается в прямую сумму неприводимых $M_{n}(D)$-модулей, каждый из которых является линейньгм пространством над $\mathbb{Q}$ размерности $е n f^{2}$ (это хорошо известный факт из теории простых центральных алгебр). Следовательно,

$$
e n f^{2} \mid 2 d
$$

Утверждаем, что

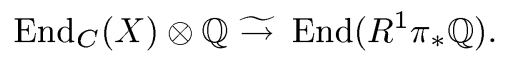

Случай $d \leqslant 3$ рассматривается в $[6,4.4 .13]$. Поэтому можно считать, что $d \geqslant 5$. Из соотношения (11.1) следует, что $f=1$. Значит, $D=Z$, и для любого вложения $\varphi: Z \hookrightarrow \mathbb{R}$ алгебра $H \otimes_{Z} \mathbb{R}_{(\varphi)}$ является матричной алгеброй над $\mathbb{R}$. Поэтому (11.2) следует из $[6,4.4 .11]$.

Заменяя $X \rightarrow C$ морфизмом $X \times{ }_{C} C^{\prime} \rightarrow C^{\prime}$ для некоторого конечного накрытия $C^{\prime} \rightarrow C$, можно считать, что

$$
K_{0}=\operatorname{End}_{C}(X) \otimes \mathbb{Q} \Im \operatorname{End}\left(R^{1} \pi_{*} \mathbb{Q}\right)
$$

В этой ситуации

$$
K_{0}=\operatorname{End}\left(R^{1} \pi_{*} \mathbb{Q}\right)=\operatorname{End}_{\pi_{1}(C, s)}\left(H^{1}\left(X_{s}, \mathbb{Q}\right)\right)=\operatorname{End}_{G}\left(H^{1}\left(X_{s}, \mathbb{Q}\right)\right)
$$

и по лемме Шура

$$
H^{1}\left(X_{s}, \mathbb{Q}\right) \otimes \overline{\mathbb{Q}} \stackrel{\rightarrow}{\rightarrow} V_{1} \oplus \cdots \oplus V_{d}
$$

- прямая сумма попарно неизоморфных $([\operatorname{Lie} G] \otimes \overline{\mathbb{Q}})$-модулей степени 2 над $\overline{\mathbb{Q}}$. Поэтому $[\operatorname{Lie} G] \otimes \overline{\mathbb{Q}}-$ полупростая алгебра Ли типа $A_{1} \times \cdots \times A_{1}=A_{1}^{d}$. 
$\mathrm{C}$ другой стороны, известно, что $\mathrm{Hg}\left(X_{0 \bar{\eta}}\right)$ - полупростая $\mathbb{Q}$-группа типа $A_{1} \times \cdots \times A_{1}=A_{1}^{d}$, поэтому выполняется геометрический аналог гипотезы Мамфорда-Тэйта [23]. В частности, гипотеза Ходжа для $X_{0 \bar{\eta}}$ эквивалентна геометрическому аналогу гипотезы Тэйта для $X_{\eta}$, где $X_{\eta}$ - общий слой $\pi$. Поскольку гипотеза Ходжа верна для всех простых абелевых многообразий простой размерности (более того,

$$
H^{2 i}\left(X_{0 \bar{\eta}}, \mathbb{Q}\right) \cap H^{i, i}\left(X_{0 \bar{\eta}}, \mathbb{C}\right)
$$

порождается классами пересечений дивизоров [30]), легко проверить, что пространство $H^{0}\left(C, R^{i} \pi_{*} \mathbb{Q}\right)$ тривиально для нечетного $i$; для четного $i$ оно порождается $\cup$-произведениями элементов из $H^{0}\left(C, R^{2} \pi_{*} \mathbb{Q}\right)$. Более того, элементы из $H^{0}\left(C, R^{2} \pi_{*} \mathbb{Q}\right)$ алгебраические (они происходят из классов дивизоров на общем слое). Поэтому $H^{0}\left(C, R^{i} \pi_{*} \mathbb{Q}\right) \otimes H^{0}\left(C, R^{i} \pi_{*} \mathbb{Q}\right)$ порождается алгебраическими классами и гипотеза $B(X)$ верна.

Если $K$ - мнимое квадратичное расширение поля $\mathbb{Q}$, то мы имеем ситуацию, рассмотренную в следствии из п. 11.7, и для любого $i \neq d$ изоморфизм

$$
\Lambda^{d+1-i}: H^{2 d+2-i}(X, \mathbb{Q}) \stackrel{\rightarrow}{\rightarrow} H^{i}(X, \mathbb{Q})
$$

является алгебраическим.

Если $[K: \mathbb{Q}]=2 d$, то $X_{0 \bar{\eta}}$ - абелево многообразие CM-типа, группа Ходжа $\mathrm{Hg}\left(X_{0 \bar{\eta}}\right)$ является тором [24], поэтому соотношение $G \hookrightarrow \operatorname{Hg}\left(X_{0 \bar{\eta}}\right)$ и полупростота $G$ дают соотношение $G=1$. Следовательно, абелева схема $\pi: X \rightarrow C$ потенциально постоянна, и $B(X)$ верна. Теорема доказана.

\section{§ 12. Вариации}

12.1. ТЕОрема. Предположим, что для некоторого натурального числа $i \geqslant 2$ существует такой алгебраический цикл $Z_{i}$ (с рациональными коэффиииентами) коразмерности $i$ на $X \times X$, что

$$
j_{*} v^{i}=\operatorname{cl}_{X \times X}\left(Z_{i}\right) \cup j_{*} 1
$$

Тогда алгебраический класс $\left[\mathrm{cl}_{X \times X}\left(Z_{i}\right)\right]_{0 i 0 i}$ индуиирует алгебраический изоморфизм

$$
H^{2}\left(C, R^{2 d-i} \pi_{*} \mathbb{Q}\right) \stackrel{\rightrightarrows}{\rightarrow} H^{0}\left(C, R^{i} \pi_{*} \mathbb{Q}\right)
$$

ДокАЗАТЕЛЬСТво. Для $u=\operatorname{cl}_{X \times X}\left(Z_{i}\right)$ имеем разложение $u=\sum u_{p q r t}$, где $u_{p q r t} \in H^{p}\left(C, R^{q} \pi_{*} \mathbb{Q}\right) \otimes H^{r}\left(C, R^{t} \pi_{*} \mathbb{Q}\right)$. В силу леммы из п. $3.3 u_{0 i 0 i}-$ алгебраический класс.

У тверждаем, что $u_{0 i 0 i}$ индуцирует алгебраический изоморфизм

$$
H^{2}\left(C, R^{2 d-i} \pi_{*} \mathbb{Q}\right) \Im H^{0}\left(C, R^{i} \pi_{*} \mathbb{Q}\right)
$$


Действительно, рассмотрим композицию

$$
\begin{aligned}
& H^{2}\left(C, R^{2 d-i} \pi_{*} \mathbb{Q}\right) \stackrel{\mathrm{pr}_{1}^{*}}{\rightarrow} H^{2}\left(C, R^{2 d-i} \pi_{*} \mathbb{Q}\right) \otimes H^{0}(C, \mathbb{Q}) \\
& \stackrel{\cup u_{0 i 0 i}}{-} \rightarrow H^{2}\left(C, R^{2 d} \pi_{*} \mathbb{Q}\right) \otimes H^{0}\left(C, R^{i} \pi_{*} \mathbb{Q}\right) \\
& =H^{2 d+2}(X, \mathbb{Q}) \otimes H^{0}\left(C, R^{i} \pi_{*} \mathbb{Q}\right) \\
& \stackrel{\mathrm{pr}_{2 *}}{\longrightarrow} H^{0}\left(C, R^{i} \pi_{*} \mathbb{Q}\right) \text {. }
\end{aligned}
$$

В силу (6.2) алгебраический класс $j_{*} v^{i}$ индуцирует алгебраический изоморфизм

$$
H^{2}\left(C, R^{2 d-i} \pi_{*} \mathbb{Q}\right) \stackrel{\leftrightarrows}{\rightarrow} H^{2}\left(C, R^{i} \pi_{*} \mathbb{Q}\right) .
$$

Поэтому из (7.3) следуют соотношения

$$
\operatorname{dim} H^{2}\left(C, R^{2 d-i} \pi_{*} \mathbb{Q}\right)=\operatorname{dim} H^{2}\left(C, R^{i} \pi_{*} \mathbb{Q}\right)=\operatorname{dim} H^{0}\left(C, R^{i} \pi_{*} \mathbb{Q}\right),
$$

и остается доказать инъективность отображения

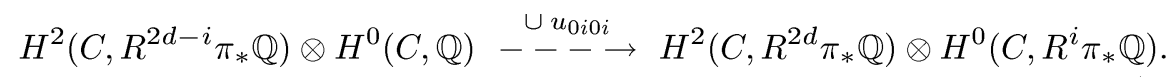

Имеем

$$
\operatorname{cl}_{X \times X}\left(Z_{i}\right)=u=u_{0 i 0 i}+\sum_{p \neq 0} u_{p q r t}+\sum_{r \neq 0} u_{0 q r t}+\sum_{q \neq i} u_{0 q 0 t} .
$$

Поскольку $\sum_{p \neq 0} u_{p q r t}$ аннулирует $H^{2}\left(C, R^{2 d-i} \pi_{*} \mathbb{Q}\right) \otimes H^{0}(C, \mathbb{Q})$, то изоморфизм (12.1) определен соответствием

$$
\left[u_{0 i 0 i}+\sum_{r \neq 0} u_{0 q r t}+\sum_{q \neq i} u_{0 q 0 t}\right] \cup j_{*} 1 .
$$

Согласно следствию из п. 8.2

$$
\begin{gathered}
j_{*} 1=\operatorname{cl}_{X \times X}\left(X \times_{C} X\right) \in\left[H^{0}(C, \mathbb{Q}) \otimes H^{2}(C, \mathbb{Q})\right] \oplus\left[H^{2}(C, \mathbb{Q}) \otimes H^{0}(C, \mathbb{Q})\right] \\
\oplus\left[H^{1}(C, \mathbb{Q}) \otimes H^{1}(C, \mathbb{Q})\right] .
\end{gathered}
$$

Поэтому $\sum_{r \neq 0} u_{0 q r t} \cup j_{*} 1$ аннулирует $H^{2}\left(C, R^{2 d-i} \pi_{*} \mathbb{Q}\right) \otimes H^{0}(C, \mathbb{Q})$, и изоморфизм (12.1) определен соответствием

$$
\left[u_{0 i 0 i}+\sum_{q \neq i} u_{0 q 0 t}\right] \cup j_{*} 1 .
$$

Наконец,

$$
\operatorname{pr}_{2 *}\left(\left[H^{2}\left(C, R^{2 d-i} \pi_{*} \mathbb{Q}\right) \otimes H^{0}(C, \mathbb{Q})\right] \cup\left[\sum_{q \neq i} u_{0 q 0 t} \cup j_{*} 1\right]\right)=(0),
$$

поэтому изоморфизм (12.1) определен алгебраическим соответствием $u_{0 i 0 i} \cup j_{*} 1$. В частности, отображение

$$
H^{2}\left(C, R^{2 d-i} \pi_{*} \mathbb{Q}\right) \otimes H^{0}(C, \mathbb{Q}) \stackrel{\cup u_{0 i 0 i} \cup j_{*} 1}{-} \underset{\longrightarrow}{\longrightarrow} H^{2}\left(C, R^{2 d} \pi_{*} \mathbb{Q}\right) \otimes H^{2}\left(C, R^{i} \pi_{*} \mathbb{Q}\right)
$$

инъективно. Поэтому отображение (12.2) инъективно. Теорема доказана. 
12.2. При выполнении условия теоремы из п. 12.1 диаграмму (7.4) можно преобразовать в новую диаграмму

$$
\begin{array}{ccccc}
H^{0}\left(C, R^{2 d+2-i} \pi_{*} \mathbb{Q}\right) & \oplus & H^{1}\left(C, R^{2 d+1-i} \pi_{*} \mathbb{Q}\right) & \oplus & H^{2}\left(C, R^{2 d-i} \pi_{*} \mathbb{Q}\right) \\
\simeq \downarrow^{\varphi_{i}} & & \simeq \downarrow^{\left[j_{*} v^{i-1}\right]^{*}} & & \simeq \downarrow^{\left[u_{0 i 0 i}\right]^{*}} \\
H^{2}\left(C, R^{i-2} \pi_{*} \mathbb{Q}\right) & \oplus & H^{1}\left(C, R^{i-1} \pi_{*} \mathbb{Q}\right) & \oplus & H^{0}\left(C, R^{i} \pi_{*} \mathbb{Q}\right)
\end{array}
$$

где $u=\operatorname{cl}_{X \times X}\left(Z_{i}\right)$ и все вертикальные стрелки являются алгебраическими изоморфизмами. Мы приходим к следуюшему результату.

12.3. Теорема. Пусть $\pi: X \rightarrow C$ - комплексная абелева схема с главной поляризацией относительной размерности d над гладкой проективной кривой С. Предположим, что для некоторого натурального числа $i \geqslant 2$ существует такой алгебраический цикл $Z_{i}$ (с рачиональньми коэффициентами) коразмерности $i$ на $X \times X$, ито

$$
j_{*} v^{i}=\operatorname{cl}_{X \times X}\left(Z_{i}\right) \cup j_{*} 1 .
$$

Пусть $u=\operatorname{cl}_{X \times X}\left(Z_{i}\right)$, и пусть $u_{0 i 0 i} \in H^{0}\left(C, R^{i} \pi_{*} \mathbb{Q}\right) \otimes H^{0}\left(C, R^{i} \pi_{*} \mathbb{Q}\right)-$ coomветствующая компонента $u$.

Тогда алгебрачческий класс

$$
\left[\Delta_{*} \mathrm{cl}_{X}\left(X_{s}\right)\right] \circ\left[j_{*} v^{i-2}\right]+j_{*} v^{i-1}+u_{0 i 0 i}
$$

определяет алгебрачческий изоморфизм

$$
H^{2 d+2-i}(X, \mathbb{Q}) \stackrel{\rightarrow}{\rightarrow} H^{i}(X, \mathbb{Q})
$$

В частности, гипотеза $B^{i}(X)$ верна.

\section{Список литературы}

1. André Y. Pour une théorie inconditionnelle des motifs // Publ. Math. IHES. 1996. V. 83. P. $5-49$.

2. Аракелов С. Ю. Семейства алгебраических кривых с фиксированными вырождениями // Изв. АН СССР. Сер. матем. 1971. Т. 35. № 6. С. 1269-1293.

3. Бурбаки Н. Группы и алгебры Ли. М.: Мир, 1972, 1976, 1978.

4. Чәжәнь Ш.-Ш. Комплексные многообразия. М.: ИЛ, 1961.

5. Deligne $P$. Théorème de Lefschetz et critères de dégénérescence de suites spectrales // Publ. Math. IHES. 1968. V. 35. P. 259-278.

6. Deligne P. Théorie de Hodge. II // Publ. Math. IHES. 1971. V. 40. P. 5-57.

7. Deligne P. La conjecture de Weil pour les surfaces K3 // Invent. Math. 1972. V. 15. P. 206-226.

8. Faltings G., Chai Ch.-L. Degeneration of Abelian varieties // Ergebnisse der Mathematik und ihrer Grenzgebiete (3). V. 22. Berlin-Heidelberg-N. Y.-London-Paris-Tokyo-Hong Kong-Barselona: Springer-Verlag, 1990.

9. Fulton W. Intersection theory. Berlin-Heidelberg-N. Y.-Tokyo: Springer-Verlag, 1984.

10. Gordon B. B. A survey of the Hodge conjecture for Abelian varieties // J. D. Lewis. A survey of the Hodge conjecture. Second edition. CRM Monograph Series. V. 10. Centre de Recherches Mathématiques Université de Montréal, 1999. P. 297-356.

11. Гриффитс Ф., Харрис Дж. Принципы алгебраической геометрии. М.: Мир, 1982. 
12. Grothendieck A. Standard conjectures on algebraic cycles // Algebr. Geom. London: Oxford Univ. Press, 1969. P. 193-199.

13. Hodge $W$. V. D. The topological invariants of algebraic varieties // Proc. Int. Congr. Math. (Cambridge, MA, 1950). V. 1. Providence: Amer. Math. Soc., 1952. P. 182-192.

14. Kleiman S. L. Algebraic cycles and the Weil conjectures // Dix exposés sur la cohomologie des schémas. Amsterdam: North-Holland, 1968. P. 359-386.

15. Kleiman S. L. The standard conjectures // Proc. Symp. Pure Math. 1994. V. 55. Part I. P. 3-20.

16. Lang $S$. Abelian varieties. N. Y.: Interscience, 1959.

17. Lang S. Fundamentals of Diophantine geometry. N. Y.-Berlin-Heidelberg-Tokyo: Springer-Verlag, 1983.

18. Lange H., Birkenhake C. Complex Abelian varieties // Grundlehren der Mathematischen Wissenschaften. V. 302. Berlin: Springer-Verlag, 1992.

19. Lieberman D. Numerical and homological equivalence of algebraic cycles on Hodge manifolds // Amer. J. Math. 1968. V. 90. P. 366-374.

20. Манин Ю. И. Соответствия, мотивы и моноидальные преобразования // Матем. сб. 1968. T. $77(119)$. С. $475-507$.

21. Милн Дж. Этальные когомологии. М.: Мир, 1983.

22. Milne J. S. Lefschetz motives and the Tate conjecture // Compositio Math. 1999. V. 117. P. $45-76$.

23. Mumford D. Families of abelian varieties // Algebraic Groups and Discontinuous Subgroups. Proc. Sympos. Pure Math. V. 9. Providence: Amer. Math. Soc., 1966. P. 347-352.

24. Mumford D. A note on Shimura's paper "Discontinuous groups and abelian varieties" // Math. Ann. 1969. V. 181. № 4. P. 345-351.

25. Pohlmann H. J. Algebraic cycles on abelian varieties of complex multiplication type // Ann. Math. 1968. V. 88. № 1. P. 161-180.

26. Серр Ж.-П. Алгебраические группы и поля классов. М.: Мир, 1968.

27. Shioda T. What is known about the Hodge conjecture? // Adv. Stud. Pure Math. 1983. V. 1. P. $55-68$.

28. Танкеев C. Г. Об алгебраических циклах на поверхностях и абелевых многообразиях // Изв. АН СССР. Сер. матем. 1981. Т. 45. № 2. С. 398-434.

29. Танкеев С. Г. Циклы на простых 5-мерных абелевых многообразиях // Изв. АН СССР. Сер. матем. 1981. Т. 45. № 4. C. 793-823.

30. Танкеев C. Г. Циклы на простых абелевых многообразиях простой размерности // Изв. АН СССР. Сер. матем. 1982. Т. 46. № 1. С. 155-170.

31. Танкеев С. Г. Циклы на абелевых многообразиях и исключительные числа // Изв. PAН. Сер. матем. 1996. Т. 60. № 2. C. 159-194.

32. Tate J. Algebraic cycles and poles of zeta functions // Arithmetical Algebraic Geometry (Proc. Conf., Purdue Univ., Lafayette, IN, 1963). N. Y.: Harper and Row, 1965. P. 93-110.

33. Tate $J$. Conjectures on algebraic cycles in $l$-adic cohomology // Proc. Symposia in Pure Math. 1994. V. 55. Part 1. P. 71-83.

34. Зархин Ю. Г. Веса простых алгебр Ли в когомологиях алгебраических многообразий // Изв. АН СССР. Сер. матем. 1984. Т. 48. № 2. С. 264-304.

Владимирский государственный университет

E-mail: tankeev-m2@vpti.vladimir.su 